University of New Hampshire

University of New Hampshire Scholars' Repository

Doctoral Dissertations

Student Scholarship

Fall 1980

\title{
TOPICS IN QUANTUM FIELD THEORY
}

CHARLES HENRY TABB

Follow this and additional works at: https://scholars.unh.edu/dissertation

\section{Recommended Citation}

TABB, CHARLES HENRY, "TOPICS IN QUANTUM FIELD THEORY" (1980). Doctoral Dissertations. 1273.

https://scholars.unh.edu/dissertation/1273

This Dissertation is brought to you for free and open access by the Student Scholarship at University of New Hampshire Scholars' Repository. It has been accepted for inclusion in Doctoral Dissertations by an authorized administrator of University of New Hampshire Scholars' Repository. For more information, please contact Scholarly.Communication@unh.edu. 


\section{INFORMATION TO USERS}

This was produced from a copy of a document sent to us for microfilming. While the most advanced technological means to photograph and reproduce this document have been used, the quality is heavily dependent upon the quality of the material submitted.

The following explanation of techniques is provided to help you understand markings or notations which may appear on this reproduction.

1. The sign or "target" for pages apparently lacking from the document photographed is "Missing Page(s)". If it was possible to obtain the missing page(s) or section, they are spliced into the film along with adjacent pages. This may have necessitated cutting through an image and duplicating adjacent pages to assure you of complete continuity.

2. When an image on the film is obliterated with a round black mark it is an indication that the film inspector noticed either blurred copy because of movement during exposure, or duplicate copy. Unless we meant to delete copyrighted materials that should not have been filmed, you will find a good image of the page in the adjacent frame.

3. When a map, drawing or chart, etc., is part of the material being photographed the photographer has followed a definite method in "sectioning" the material. It is customary to begin filming at the upper left hand corner of a large sheet and to continue from left to right in equal sections with small overlaps. If necessary, sectioning is continued again-beginning below the first row and continuing on until complete.

4. For any illustrations that cannot be reproduced satisfactorily by xerography, photographic prints can be purchased at additional cost and tipped into your xerographic copy. Requests can be made to our Dissertations Customer Services Department.

5. Some pages in any document may have indistinct print. In all cases we have filmed the best available copy.

\section{University Microfilms International}


TABB, ChaRLES HENRY

TOPICS IN QUANTUM FIELD THEORY

University of New Hampshire

PH.D.

1980

University

Microfilms

International $300 \mathrm{~N}$. Zeeb Road, Ann Arbor, MI 48106 


\title{
$0.8 \times a n c h$
}

TOPICS IN QUANTUM FIELD THEORY

BY

Charles Henry Tabb

M.A., Yeshiva University, 1975

B.S., University of Rochester, 1971

\section{DISSERTATION}

\section{Submitted to the University of New Hampshire in Partial Fulfiliment of \\ the Requirements for the Degree of}

\author{
Doctor of Philosophy \\ in \\ Physics
}

September 1980 
This dissertation has been examined and approved. Bane G. Hamilton
Dissertation director Barry J. Harrington
Associate Professor of Physics

Harvey K. Shepard 8/7/80

Dissertation director, Harvey K. Shepard Professor of Physics
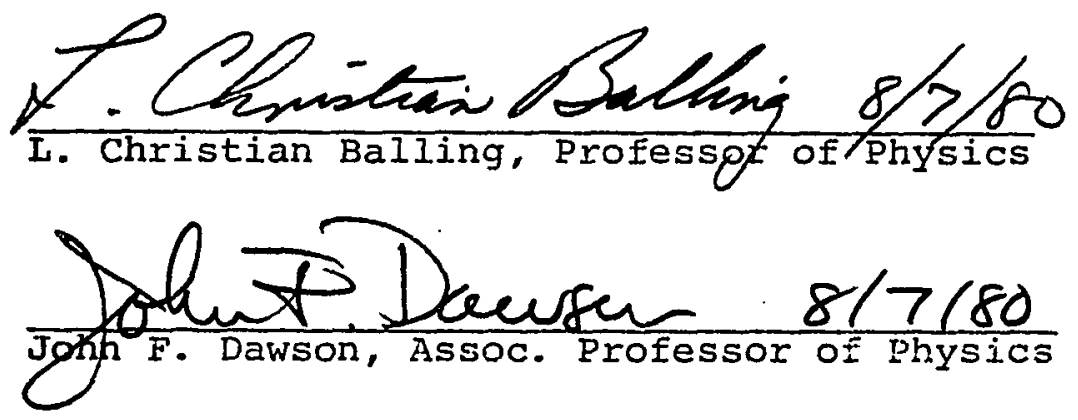

forked heisenberg

Jochen Heisenberg, Assoc. Professor of Physics

August 7,1980 
DEDICATION

To Randi and Jamie 


\section{ACKNOWLEDGEMENTS}

Among the many people to whom I am endebted, the following deserve special acknowledgement.

First and foremost, I would like to thank my wife, Randi, for her patience and support these many years. I would like to thank Barry J. Harrington, my friend and advisor, for devoting many long hours to patiently teaching, explaining, discussing, and working with me. I want to express special thanks to Harvey Shepard for his support along with many comments and suggestions and for his critical reading of the manuscript.

I am also indebted to sue Horner and Professor Len Fisk for all of their creative endeavors on my behalf. I would like to thank John Dawson for all of his help this past year.

Many thanks go to Dean Richard Davis, Professor John Lockwood, Dean Raymond Erickson, and Dean William Drew for their part in my financial support.

I would like to thank Andy Strominger, Sudip Chakravarty, John Richardson, and Lee Smolin for many stimulating conversations and everyone at the Institute for Theoretical Physics at the University of California, Santa Barbara, for their hospitality.

Thanks also go to sue Hamel and Laurie Ferris for typing the manuscript. 
I am greatly indebted to Pauline Fox Podgor for all of her generous support.

Lastly, I would like to thank my parents, Morris and Rose Tabb for all the encouragement they have given me. 
PREFACE

\section{Unification}

The primary goal of particle physics today is to achieve Einstein's dream of finding a unified description of nature at the microscopic level. Although Einstein was unsuccessful in unifying electromagnetism and gravity within one theory, much progress has recently been made within "Unified Field Theory". What is desired is to start with one physical principle or one symmetry of nature and with the inclusion of a minimum of experimental parameters to derive the known particle spectrum, masses of particles and force laws that describe microscopic physics. Recently the Weinberg-Salam Model of the electroweak j.nteractions has made great progress in this direction by combining the theory of electromagnetism and the weak interactions into one finite theory. Within the so-called Grand Unified Theories this work has been extended to also include Quantum Chrmodynamics (Q.C.D.), the proposed theory of "hadron physics" or the strong interactions. Although "grand unification" is by no means complete, much has been done to suggest that this method of unification is indeed viable. Efforts to include gravitation within a unified theory have still been most frustrating. The canonical theory of Quantum Gravity is plagued with divergences which cannot be renormalized away. (This is similar to the situation in weak interaction physics before the advent of the Weinberg-salam 
Model.) However, impressive results have lately been found in supergravity theory which unites gravity with particle physics by postulating "supersymmetries" which encompass both fermions and bosons within one symmetry group. It appears, however, that supergravity theories predict a somewhat different particle spectrum than what is currently considered to be the fundamental particles of nature. Another attempt at unification, perhaps more in keeping with the Weinberg-Salam Model, is to construct a theory possessing a local scale invariance which at low energies can be modeled by an effective theory which looks like Einstein's theory of gravitation. A totally different line of thought exists among many relativists who feel that the union of quantum mechanics and relativity within quantum field theory is unnatural and must be replaced by a more fundamental theory at the small distance scale of quantum gravity.

Except for this latter point of view, all the attempts at unification have one problem in common. In order for these theories to "spontaneously break" to give the known physics at the relatively low energies presently accessible to experiment, there must exist fundamental scalar particles called Higgs particles. No such particles have yet been found in nature, and it has been argued that symmetry breaking using such particles leads to "unnatural" renormalization. When this happens the occurence of very different energy scales in the theory causes very small fluctuations in certain "bare" parameters to force very large changes in 
some renormalized parameters. Thus there is no simple way to make order of magnitude estimates of the "physical" renormalized parameters.

Instead of Higgs particles, it is felt by many that what should occur is a much more complex process called dynamical symmetry breaking in which the Higgs particle is replaced by a composite fermion-antifermion pair. (This is the case for Cooper Pairs in superconductivity.) Within the Grand Unified Theories one approach to the composite problem has been to postulate that the composite field is made of fundamental spin $\frac{1}{2}$ fields possessing a new quantum number dubbed technicolor, and that the composite is a technicolor singlet. (It is technicolor neutral.)

The Grand Unified Theories give way to the physics at presently accessible energies as follows. First, a large symmetry group is proposed. This symmetry allows for the existence of all the known fundamental particles as well as others. At very high energy all of these particles would exist and the symmetry would be intact. At lower energies the symmetry would be broken, but in such a way that various subgroups of symmetries would remain unbroken. When symmetries break, certain particles in the theory acquire a mass. In this way the original symmetry would give way to a subset of symmetries and hopefully to the observed particle spectrum. Among the particles predicted in this fashion are particles of such large mass that they have not yet been found. 


$$
\text { Non-Abelian Gauge Theory -- Q.C.D. }
$$

The Weinberg-Salam theory, Q.C.D., and Einstein's theory of gravitation all fall within a class known as non-abelian gauge theories. The fact that all of these theories are of the same type makes unification much more plausible. The character of a theory possessing a non-abelian symmetry as opposed to an abelian symmetry such as Quantum Electrodynamics (Q.E.D.) is such that in the non-abelian case the group generators do not commute, whereas in the abelian case they do. This corresponds to the fact that in the abelian case (Q.E.D.) the photon field does not carry a charge, while in the non-abelian case the field is charged and hence selfinteracting. This leads to nonlinearity of the field equations for the pure non-abelian case. Because of these non-linearities there is much more possibility for rich structure within these theories, and indeed, various nonperturbative structures such as instantons, solitons, etc., have been discovered. Unfortunately, these non-linear theories lead to very difficult mathematics and hence to the existence of many unresolved problems.

The unified theories are postulated to break down to Q.C.D. and the Weinberg-Salam theory at currently accessible energies. Q.C.D. is a vector gauge theory with symmetry group SU(3). The non-abelian charge is named "color charge" and the eight types of "colored" vector particles (gluons) carry the strong interaction between three types of "colored" spinor particles called quarks. 
Q.C.D. has a property known as asymptotic freedom. At very short distances (or very high energies), there is very little self-interaction of the gluon field, and the strong force becomes very weak, thus allowing perturbative approximations. At low energies (the energy of a hadron or approximately $\left.10^{2}-10^{3} \mathrm{MeV}\right)$, the quarks are far apart and there is much more self interaction. The strong force is then very strong, and the perturbative approximation breaks down. This phenomenum is known as infrared slavery. It is believed that the strong force becomes so strong that all colored field Iines are trapped or "confined" at interquark distances greater than and of the order of one fermi $\left(10^{-13} \mathrm{~cm}\right)$. If this is the case, then all "physical" particles are color singlets, or neutral, with respect to the color charge. A colored particle (quark or gluon) could then only exist in conjunction with other colored particles in such a way that the total color charge is zero. This phenomenum is called color confinement.

Within the quark model, the known hadrons are supposed to be made of confined quarks. A baryon (proton, neutron, etc.) is made of three quarks, while a meson (pion, kaon, etc.) is made of'a quark-antiquark pair. A problem is that it has only been conjectured that quarks are confined. Because of the non-perturbative nature of confinement it has not yet been demonstrated as a natural consequence of Q.C.D. 


\section{Confinement}

There have been various attempts to get around the infrared problem in Q.C.D. The "Bag" Model ensures confinement by placing the quarks in a cavity with imposed boundary conditions. Other approaches attempt to calculate infrared effects by modifying the original theory to include only a subset of the original Feynman diagrams. Among these approximations is the $\frac{1}{N}$ expansion which takes the limit of $N$ (the number of colors) as very large and uses $\frac{1}{\hat{N}}$ as an expansion parameter. There is also a current program which only includes the longitudinal part of vertex functions within the infrared region of the theory.

One practical approach to calculating within the strong coupling sector of the theory is that of Lattice Gauge Theory. In this work, space-time is put on a lattice and into a finite size "box". Then calculations can be done using various Monte-Carlo techniques. These lattice methods provide evidence that confinement exists within the lattice theory of Q.C.D., and also that there is no phase transition within the lattice theory. That is, at high energies quarks will still be confined.

The latter two methods give results in agreement with a "string" model of confinement, in that the color confining potential is linear in the distance between the confined quarks. This helps to justify the non-relativistic linear potential models for the Charmonium and Upsilon states. 
other suggested avenues of investigation for the confinement problem include the study of topological structure in Q.C.D. One example of this is the "Gribov Problem" whereby the topology of Q.C.D. does not lend itself to unambiguously fixing the gauge degrees of freedom. Also the existence of "Renormalon" singularities in the Borel summed perturbation series may be intimately related to the confinement problem.

Currently, a popular hypothesis is that the confining mechanism may be a property of a non-trivial field vacuum. Within this approach a so-called background field configuration or non-zero vacuum expectation value of the vector field is assumed, and a perturbation theory is built around it. Examples of this method include expansions around finite action or instanton solutions which do indicate structures that may be linked with confinement. These calculations, however, appear to break down within the energy range of interest for confinement.

Another model for the Q.C.D. vacuum is the color magnetic vortex condensate model also called the Copenhagen Model. This is a condensate of color magnetic flux tubes. It is thought that quantum fluctuations within this model will cause the flux tubes to orient themselves in random directions, producing rotational invariance and thus forming a quantum liquid which will confine. Both of the latter two models are still undergoing active investigation. 


\section{Vacuum structure}

The vacuum of quantum field theory can accommodate structures not usually associated with "empty space". This structure is responsible for symmetry breaking, both spontaneous and dynamic, and hopefully for confinement. In quantum field theory, the first notion of vacuum structure is the infinite zero point energy (the zero point energy of an infinite number of harmonic oscillations.) This infinite energy is discarded in the interaction picture when the theory is expanded perturbatively around the trivial (zero field) background. It may be, however, that the lowest energy state of the theory is not the zero field state, but a state in which the quantum field gets a non-zero vacuum expectation value. This is analogous to cases in many-body theory in which a Bose-condensate is formed. Spontaneous symmetry breaking occurs when the classical potential is such that the state with a non-zero expectation value for the Higgs field is favored energetically. In this case the classical action of the theory is invariant to a certain symmetry, but because the quantum theory is built upon a non-symmetric background, the symmetry is broken. To generate symmetry breaking without fundamental scalars, a composite fermion-antifermion field must acquire a non-zero vacuum expectation value. In order for this to happen, quantum corrections to the ground state energy within the non-abelian theory must be such that the composite field attains a non-zero expectation value within the true quantum ground state. 
An example of dynamical symmetry breaking is that of superconductivity in which the "order parameter" is the wavefunction for a Cooper pair of electrons. To this date, however, no one has been able to demonstrate how a non-zero vacuum expectation value of a composite fermion-antifermion field is generated within the non-abelian theories.

A favorite model of confinement is also in analogy to effects in superconductivity. Within the Type II superconducting ground state, magnetic flux will be quantized into strings. If magnetic monopoles could be introduced, these strings of magnetic flux would confine a monopoleantimonopole pair.

It is thought that the Q.C.D. vacuum is in some sense dual to the superconducting state. Perhaps we have a condensate of color magnetic monopoles causing confinement of color electric charges (quarks). It has been suggested that the color magnetic vortices or flux tubes within the Copenhagen model are the objects dual to the cooper pair fields in superconductivity and thus that the copenhagen vacuum should confine quarks.

\section{Gauge Invariance and the Effective Action}

A problem peculiar to gauge theories is that all physical quantities must be invariant to the choice of gauge. Within abelian theories such as Q.E.D., the electric and magnetic fields are gauge invariant objects and thus physical, while the vector potential is not. Within non-abelian theories such as Q.C.D., the color electric and magnetic fields are not 
gauge invariant and the problem of defining physical quantities is much more complicated. Another direction of study for many of the problems that have been outlined above is a formulation of gauge theory that does not take the vector potential or electromagnetic field as the fundamental object. Instead, an attempt is made to construct the theory from physical, gauge invariant, non-local operators. We will not pursue this study here.

In order to study the quantum structure of a field theory expanded around a non-zero background field, we define the Effective Action Functional. This quantity is a functional of the background field which reduces to the classical action in the "tree" (classical) approximation. To study the stability of the gauge theory vacuum, we demand that the effective action be gauge invariant. Up until recently, however, the popular definition of the effective action for gauge theories was not gauge invariant. In the text we shall formally investigate the derivation and properties of a gauge invariant definition of the effective action. We shall then use the invariant definition in a semi-classical study of Q.C.D. expanded around a non-zero background field. In particular we shall investigate effects due to the presence of a constant color magnetic background field. These effects and their interpretation are of fundamental importance in the construction of the copenhagen flux tube model of the Q.C.D. vacuum. 
TABLE OF CONTENTS

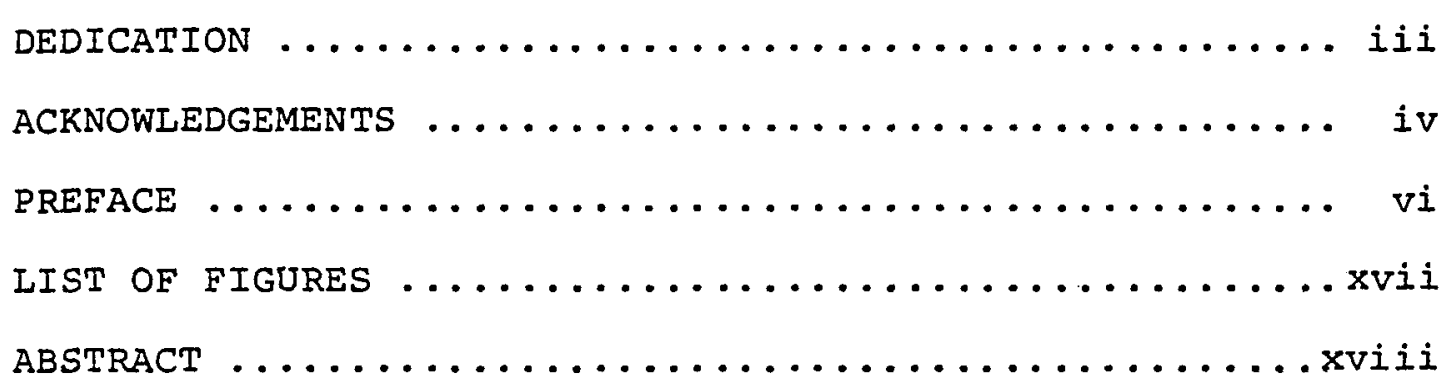

I. INTRODUCTION $\ldots \ldots \ldots \ldots \ldots \ldots \ldots \ldots \ldots \ldots \ldots \ldots \ldots \ldots \ldots \ldots$

II. GAUGE INVARIANT ACTION FUNCTIONAL ........... 6

A. The Effective Action ................. 6

B. Gauge Invariance ................... 13

III. THE CONSTANT MAGNETIC FIELD AND ZERO POINT ENERGY 17

IV. NEGATIVE EIGENVALUES AND InStability ........ 35

V. DISCUSSION AND SUMMARY ................ 53

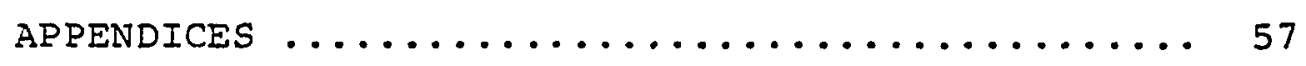

Appendix A: Comparison of Classical

Minimality and Positive Definiteness of

the One-Loop Eigenvalues for Yang-Mills

Fields ........................ 58

Appendix B: Gyroscopic Stability .........66 63

IIST OF REFERENCES $\ldots \ldots \ldots \ldots \ldots \ldots \ldots \ldots \ldots \ldots \ldots$ 


\section{LIST OF FIGURES}

Figure 1. Quartic Potential, $V(\phi)$, with a negative mass squared versus $\phi . \ldots \ldots \ldots \ldots \ldots \ldots \ldots \ldots \ldots \ldots \ldots \ldots \ldots \ldots \ldots$

Figure 2. Integrand of minus the Euclidean action versus the field modes, $\phi_{k}$, for various values of $k^{2} \ldots \ldots \ldots \ldots . \ldots 76$

Figure 3. Quadratic approximations to the integrands which appear in Figure $2 \ldots \ldots \ldots \ldots \ldots \ldots \ldots \ldots \ldots \ldots$

Figure 4. Solid lines-Euclidean functional integrand for $\mathrm{k}^{2}=0$ versus field modes, $\phi$, for $\mathrm{m}_{I I}^{\prime}>\mathrm{m}_{I}^{\prime}$. Dashed lines--Quadratic approximation to the solid lines where $m_{I I}=m_{I I}^{\prime}, m_{I}=m_{I}^{\prime} \ldots \ldots \ldots \ldots \ldots \ldots \ldots \ldots \ldots \ldots \ldots . \ldots \ldots$

Figure 5a. Euclidean functional integrand for $k^{2}=0$ and positive mass squared in the complex field mode plane...... 79

Figure 5b. Same as in Figure 5b except for negative mass squared................................. 79

Figure 6. Analytic continuation of the functional integrand represented in Figure 3 for various values of $\mathrm{k}^{2}<\mathrm{m}^{2}$ where

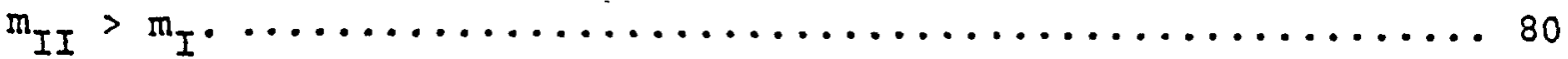




\section{ABSTRACT}

TOPICS IN QUANTUM FIELD THEORY

by

Charles Henry Tabb

University of New Hampshire, August, 1980

\section{The Abstract}

A gauge invariant action functional for non-abelian gauge theories is derived from the vacuum to vacuum transition amplitude. This effective action is then examined in the loop expansion, where it is demonstrated that the unrenormalized one-loop term is equal to the change in the zero point energy of the theory due to the presence of a non-vanishing background field. The one loop term is carefully evaluated in Euclidean space, and the positivity of the one-loop eigenvalues is related to classical stability in terms of energy minimality. The case of regative eigenvalues is considered and a procedure for "regularizing" the one loop term for this problem is defined. An imaginary part of the effective action to this order is shown to indicate an instability of the theory in the presence of a non-vanishing background field. Finally, complications due to the occurrence of "gyroscopic" terms are reviewed. 


\section{INTRODUCTION}

The effective action functional, $\Gamma\left(\phi_{i}\right)$, of a quantum field theory is commonly used to study symmetry properties. It is widely used in a semiclassical (100p) approximation to study the effect of quantum phenomena on certain classical symmetries and hence is extremely useful in the study of spontaneous symmetry breakdown. ${ }^{1}$ The effective action, like the classical action, may also be used to study the dynamics of the theory. It is a generator of Greens functions and has been used in the study of vacuum stability. In this regard, it will hopefully be useful in the study of confinement in Quantum Chromodynamics, where a non-trivial vacuum configuration may be responsible for color confinement. 2 There are a number of definitions of $\Gamma$ in the literature. ${ }^{1,3-8}$ we will define $\Gamma$ as the generator of one-particleirreducible Greens functions. 1,5 It has been shown for non-gauge theories that this definition is such that ${ }^{3}$

$$
\left\langle 0\left|\frac{\delta S}{\delta \Phi_{i}}\right| 0\right\rangle=\frac{\delta \Gamma}{\delta \phi_{i}}
$$

where " $i$ " runs over all indices of the theory including those of space-time, $S\left(\Phi_{i}\right)$ is the classical action, $\Phi_{i}$ is the operator field and

$$
\phi_{i}=\left\langle 0\left|\Phi_{i}\right| 0\right\rangle
$$


i.e., $\phi_{i}$ is the vacuum expectation value of the quantum field. With this definition, we can also show that

$$
\frac{\delta \Gamma}{\delta \phi_{i}}=-J_{i}
$$

in analogy with the classical equations of motion, i.e., $J_{i}\left(\phi_{i}\right)$ is an external source coupled linearly to the fields, and also

$$
\frac{\delta^{2} \Gamma}{\delta \phi_{i} \delta \phi_{j}} G^{j k}=-\delta_{i}^{k}
$$

where $G^{j k}$ is the Feynman propagator of the theory. The higher functional derivatives of $\Gamma$ are the full irreducible vertex functions of the theory, whereas the functional derivatives of the classical action give the bare vertex functions. 3,4 In the tree approximation, the effective action $\Gamma\left(\phi_{i}\right)$ reduces to the classical action $s\left(\phi_{i}\right)$.

$\Gamma$ is usually derived from the vacuum to vacuum transition amplitude: ${ }^{1,3-5}$

$$
Z(J)=\left\langle 0^{+} \mid 0^{-}\right\rangle^{J}
$$

where

$$
Z(J)=\exp [i W(J)]
$$


and $W(J)$ is the generating functional for connected Greens functions. Now $\Gamma$ is defined by the Legendre transformation:

$$
\Gamma\left(\phi_{i}\right)=W\left(J_{i}\right)-J_{i} \phi_{i}
$$

where

$$
\phi_{i}=\frac{\delta W}{\delta J_{i}} .
$$

If the above definitions are extended to gauge theories, $\Gamma\left(A_{\mu}\right)$ is, in general, not gauge invariant [there is some controversy over this point in the literature]. ${ }^{9}$ Renormalization procedures needed to regularize $\Gamma$ will also be gauge dependent, although physical processes should still maintain the gauge invariance of the theory. Recently, a number of alternative functionals have been proposed in order to define a gauge invariant functional from which we could give a gauge invariant renormalization scheme.10-14 These employ the so-called background field methods and gauges. 't Hooft ${ }^{15}$ defines such a functional. We will show in Section 2 that this functional can be derived from the usual definition of the effective action functional by way of a Legendre transformation. We will then explicitly demonstrate its gauge invariance properties.

our interest in the above study has been piqued by recent developments in the literature. In an attempt to 
discover a model for the non-trivial vacuum of $Q C D$, several authors have investigated the one-loop approximation to the effective action in the presence of a constant color magnetic field $\mathrm{B}_{\mathrm{a}}^{\mathrm{i}}$. $^{16-23}$ Such a configuration provides an effective infrared cut-off for the theory, although renormalization group calculations cannot be readily extended to small values of $B$. For such small values of $B$, the one-loop term lowers the field energy and, although the range of applicability of this calculation is unclear, it has been surmised that a constant color magnetic field leads to a lower ground state energy than the zero field (perturbative) ground state energy. Nielsen and olesen 16 calculate this one-loop effective action by extending the result that the one-loop effective action for non-gauge theories in the presence of a non-zero constant background field can be written as the change in the zero point energy of the theory due to the background field. 24-26 In section 3 , we verify that this result can be extended to gauge theories with $\Gamma$ defined as in section 2 and to theories with spatially dependent background fields and we demonstrate under what conditions it will presumably break down.

The calculation of the effective action in the presence of a constant color magnetic background field also leads to an imaginary part of the effective action $16,18,22$ in analogy with the effective action for a constant electric field in 
QED. ${ }^{6}$ This has been interpreted as an indication of an instability of the theory when expanded around a constant B field, and Nielsen and Olesen proceed to search for other field configurations which lead to even lower energies and a stable vacuum. 27-31 In section 4, we investigate the field modes responsible for this imaginary part by examining the Euclidean version of the theory. We point out some ambiguities in the definition of the imaginary part when looked at from this point of view. By going to Euclidean space we are also able to make analogies with the classical stability analysis and we conclude that this instability is actually of classical origin although it appears in the first quantum correction. (Indeed, the classical Yang-Mills theory in the presence of a constant magnetic field has shown to be unstable. 32,35 , we compare this type of instability with those of quantum origin pointed out by Callan and coleman ${ }^{1,36-38}$ and with those due to pair production pointed out by schwinger. ${ }^{6}$ In analogy with the last example we show under what conditions the imaginary part can be used to calculate a decay rate and under what conditions the loop expansion is still well defined.

In Section 5, we discuss and summarize our main results. Appendix A explicitly discusses the complications which arise in trying to extend the analogy between positive definiteness of the one loop eigenvalues and classical energy minimality to Yang Mills theories.

In Appendix B, we review the problem of gyroscopic terms in the classical Hamiltonian and extend some of the previous analysis to include this case. 
II. GAUGE INVARIAIT ACTION FUNCTIONAL

\section{A. The Effective Action}

't Hooft's background field method ${ }^{15}$ reduces to an older formulation $10,12,14$ at the one-loop level. This older formulation generates reducible diagrams when we go to higher order in the loop expansion, whereas with 't Hooft's method we generate only irreducible diagrams. 't Hooft's functional is:

$$
\begin{aligned}
& \exp \frac{i}{\hbar} G(\bar{A}, J)=\int[d A(x)][d \phi(x)]\left[d \phi^{*}(x)\right] \exp \frac{i}{\hbar} \int d^{4} x\{L(\bar{A}+A)+ \\
& \left.+J_{\mu}^{a} A^{\mu a}-\frac{1}{2 \alpha}\left[D_{\mu}^{a b}(\bar{A}) A^{\mu b}\right]^{2}-\phi^{a^{*}}\left[D_{\mu}^{a c}(\bar{A}) D^{\mu c b}(\bar{A}+A)\right] \phi^{b}\right\} .
\end{aligned}
$$

A few comments are in order: $A_{\mu}^{a}(x)$ is a c-number field, representing the quantum field over which we are doing the functional integral. $\overline{\mathrm{A}}_{\mu}^{\mathrm{a}}(\mathrm{x})$ is a c-number external field around which we are expanding. $J_{\mu}^{a}=J_{\mu}^{a}(\bar{A})$ where $J_{\mu}^{a}(\bar{A})$ is to be determined by the equations of motion:

$$
\frac{\mathrm{d}}{d \bar{A}_{\mu}^{\mathrm{a}}} G[\overline{\mathrm{A}}, J(\overline{\mathrm{A}})]=\sim^{\mu \mathrm{a}}(\overline{\mathrm{A}})
$$

where the functional derivative above is meant in the sense of a total derivative rather than a partial derivative. The Lagrangian density $L$ may in general contain other sources composed of charged or Higgs particles coupled in 
a gauge invariant way. We will not consider this possibility in what follows. $\phi^{a}(x)$ and $\phi^{a *}(x)$ are ghost fields and

$$
D_{\mu}^{a b}(\bar{A})=\delta^{a b} \partial_{\mu}+c^{a c b} \bar{A}_{\mu}^{c}
$$

Also, consistent with Eq. $(10), J^{\mu a}(\bar{A})$ can be constructed so as to satisfy

$$
D_{\mu}^{a b}(\bar{A}) J^{\mu b}(\bar{A})=0
$$

Now define

$$
\Gamma(\bar{A}) \equiv G[\bar{A}, J(\bar{A})]
$$

't Hooft demonstrates that $\Gamma(\overline{\mathrm{A}})$ is indeed the generating functional of the irreducible vertices. To define the physical theory we demand that

$$
J_{\mu}^{a}(\bar{A})=0
$$

This determines $\overline{\mathrm{A}}_{\mu}^{\mathrm{a}}$ and is analogous to the classical equations of motion since

$$
\frac{d \Gamma(\bar{A})}{d \bar{A}_{\mu}^{a}}=0 .
$$


In analogy with the scalar theories, the second functional derivative of $\Gamma$ is the inverse propagator and the higher functional derivatives give the irreducible vertices.

We will now proceed to derive $\Gamma(\bar{A})$ from the vacuum to vacuum transition amplitude:

$$
\left\langle 0^{+} \mid 0^{-}\right\rangle^{J} \equiv z[J, \bar{A}(J)]=\exp \frac{i}{\hbar} W[J, \bar{A}(J)]
$$

$W[J, \bar{A}(J)]$ is the generating functional for the connected Greens functions. $z[J, \bar{A}(J)]$ can be derived from the Hamiltonian formulation of the functional integral within the A gauges ${ }^{39}$ to give:

$$
\begin{aligned}
Z[J, \bar{A}(J)]= & \int[d A] \delta\left\{x^{a}[A, \bar{A}(J)]\right\} \\
& x \operatorname{det}\left(\frac{d x^{a}}{d \theta^{b}}\right) \exp \frac{i}{\bar{n}} \int a^{4} x\left[L(A)+J_{\mu}^{a} A^{\mu a}\right]
\end{aligned}
$$

where $\overline{\mathrm{A}}_{\mu}^{\mathrm{a}}(J)$ is an as yet unspecified but invertible function of $J \cdot \chi^{a}[A, \bar{A}(J)]$ is the gauge constraint and $\operatorname{det}\left(d x^{a} / d \theta^{b}\right)$ is the appropriate Faddeev-Popov determinant. since $x^{a}$ is a cyclic variable in the sourceless theory we may freely fix $x^{a}$ so long as the theory remains well defined. We choose the so-called covariant background gauge condition: 


$$
x^{a}=D_{\mu}^{a b}(\bar{A})\left[A^{\mu b}-\bar{A}^{\mu b}\right]-E^{a} \cdot(x) .
$$

The action is invariant under inhomogeneous gauge transformations of $A_{\mu}^{a}(x)$ :

$$
A_{\mu}^{a}(x)+A_{\mu}^{a}+c^{a b c} \theta_{A}^{b} c-\partial_{\mu} \theta^{a}=A_{\mu}^{a}-D_{\mu}^{a b}(A) \theta^{b} .
$$

Therefore

$$
\frac{d x^{a}}{d \theta^{b}}=\frac{d x^{a}}{d A_{\mu}^{c}} \frac{d A_{\mu}^{c}}{d \theta^{b}}=-D_{\mu}^{a c}(\bar{A}) D^{\mu c b}(A) .
$$

We now make the change of variables $A_{\mu}^{a}+A_{\mu}^{a}+\bar{A}_{\mu}^{a}$ (J) to get

$$
\begin{aligned}
\exp \frac{i}{\hbar} W[J, \bar{A}(J)]= & \int[d A] \delta\left[D_{\mu}^{a b}(\bar{A}) A^{\mu b}-f^{a}(x)\right] \times \\
& \times \operatorname{det}\left[-D_{\mu}^{a c}(\bar{A}) D^{\mu C b}(A+\bar{A})\right] \times \\
& \times \exp \frac{i}{\bar{\hbar}} \int d^{4} x\left\{L(A+\bar{A})+J^{\mu a}\left[A_{\mu}^{a}+\bar{A}_{\mu}^{a}\right]\right\} .
\end{aligned}
$$

Let us take $w$ to be formally only a function of $J$, although as yet we still have not specified $\bar{A}_{\mu}^{a}(J)$. Then we may define $\Gamma$ by the Legendre transformation:

$$
\Gamma\left(A_{p}\right) \equiv W(J)-\int d^{4} x J_{\mu}^{a} \frac{d W}{d J_{\mu}^{a}}
$$


where we define

$$
A_{a, p}^{\mu} \equiv \frac{d w}{d J_{\mu}^{a}}
$$

as the physical field. Therefore

$$
\begin{aligned}
\exp \frac{i}{\hbar} \Gamma\left(A_{p}\right)= & \int[d A] \delta\left[D_{\mu}^{a b}(\bar{A}) A^{\mu b}-f^{a}(x)\right] \times \\
& \times \operatorname{det}\left[-D_{\mu}^{a b}(\bar{A}) D^{\mu b c}(A+\bar{A})\right] \exp \frac{i}{\bar{\hbar}} \int d^{4} x[L(A+\bar{A})+ \\
& \left.+J^{\mu a}\left(A_{p}\right) A_{\mu}^{a}+J^{\mu a}\left(A_{P}\right)\left[\bar{A}_{\mu}^{a}-A_{\mu}^{a}\right]\right\} .
\end{aligned}
$$

Thus if we choose $\overline{\mathrm{A}}_{\mu}^{a}(\mathrm{~J})$ such that

$$
\bar{A}_{\mu}^{a}(J)=A_{\mu, p}^{a}(J)=\frac{d W(J)}{d J^{\mu a}}
$$

then all the $J$ dependence at the tree level will cancel. We now use the usual exponentiation 1,40 of the gauge fixing term and Faddeev-popov determinant to give

$$
\begin{aligned}
\exp \frac{i}{\bar{z}} \Gamma(\bar{A}) & =\int[d A(x)][d \phi(x)]\left[d \phi^{*}(x)\right] \exp \frac{i}{\bar{\hbar}} \int d^{4} x\{L(\bar{A}+A)+ \\
& \left.+J_{\mu}^{a}(\bar{A}) A^{\mu a}-\frac{1}{2 \alpha}\left[D^{a b}(\bar{A}) A^{\mu b}\right]^{2}-\phi^{a *}\left[D_{\mu}^{a c}(\bar{A}) D^{\mu c b}(\bar{A}+A)\right] \phi^{b}\right\},
\end{aligned}
$$


and we have reconstructed 't Hooft's generating functional. Also, Eq. (10) follows from the Legendre transformation, Eqs. (21) and (22). Again as in the scalar case $\Gamma$ generates the one-particle-irreducible Greens functions. To the tree level $\Gamma$ is just the classical action. $\bar{A}_{\mu}^{a}(x)$, however, is no longer the vacuum expectation value of the quantum field. To see this, note that

$$
\begin{aligned}
\bar{A}_{\mu}^{a}(J) & =\frac{d W[J, \bar{A}(J)]}{d J^{\mu a}} \\
& =\frac{\delta W}{\delta J^{\mu a}}+\frac{\delta W}{\delta \bar{A}_{\nu}^{b}} \frac{d \bar{A}_{\nu}^{b}}{d J^{\mu a}}
\end{aligned}
$$

where by the partial functional derivative, $\delta$, we mean the functional derivative of $w$ with respect to only the explicit $J$ or $\bar{A}$ dependence. From the definition of $W$, Eqs. (15) and (16), we can see that

$$
\frac{\delta w}{\delta J^{\mu a}}=\frac{\left\langle 0^{+}\left|A_{\mu}^{a}\right| 0^{-}\right\rangle}{\left\langle 0^{+} \mid 0^{-}\right\rangle} \equiv\left\langle A_{\mu}^{a}\right\rangle \text {. }
$$

Therefore $\overline{\mathrm{A}}_{\mu}^{\mathrm{a}} \neq\left\langle\mathrm{A}_{\mu}^{\mathrm{a}}\right\rangle$. The distinction develops because of the dependence of the gauge condition on $\bar{A}_{\mu}^{a}$. The difference between this and the scalar theory is the implicit $J$ dependence in $x^{a}$ through its dependence on $\bar{A}_{\mu}^{a}(J)$. To see the explicit distinction, we calculate $\left\langle A_{\mu}^{a}\right\rangle$. In Eq. (26), let us change variables $A_{\mu}^{a}+A_{\mu}^{a}+\bar{A}_{\mu}^{a}$ (J) to give 


$$
\begin{aligned}
& <A_{\mu}^{a}>=\bar{A}_{\mu}^{a}(J)+ \\
& +\frac{\int[d A] A_{\mu}^{a} \delta\left[x^{a}(\bar{A}, A+\bar{A})\right] \operatorname{det}\left(\frac{d x^{a}}{d \theta^{b}}\right) \exp \frac{i}{\hbar} \int d^{4} x\left[L(A+\bar{A})-L(\bar{A})+J_{\mu^{A}}^{a} a_{1}\right.}{\int[d A] \delta\left[x^{a}\right] \operatorname{det}\left(\frac{d x^{a}}{d \theta^{b}}\right) \exp \frac{i}{\bar{h}} \int d^{4} x\left[L(A+\bar{A})-L(\bar{A})+J_{\mu}^{a} \mu a\right]} .
\end{aligned}
$$

The second term above is the expectation value of the quantum field calculated in a new "shifted" ${ }^{3}$ theory. Therefore let us write this as

$$
\left\langle A_{\mu}^{a}\right\rangle=\bar{A}_{\mu}^{a}(J)+\left\langle 0_{+}^{1}\left|A_{\mu}^{a_{\mu}}\right| 0_{-}\right\rangle /\left\langle 0_{+}^{1} \mid 0_{-}^{\prime}\right\rangle .
$$

The difference between $\left\langle A_{\mu}^{a}\right\rangle$ and $\bar{A}_{\mu}^{a}(J)$ is of order $\hbar$. In the usual formulation for scalar fields, the expectation value of the quantum field within the shifted vacuum vanishes. Here, however, in order to maintain gauge invariance (as we shall presently show) the shifted vacuum expectation value of $A_{\mu}^{a}$ does not vanish, or to put it another way, the physical field or order parameter of the theory differs from the vacuum expectation value of the quantum field. This result is similar to that of Fischler and Brout ${ }^{41}$ where they demonstrate that, to preserve gauge invariance for an abelian theory with scalar fields, the order parameter is not taken to be the naive vacuum expectation value of the scalar field. 
B. Gauge Invariance

We now proceed to demonstrate the gauge invariant properties of the effective action. If

$$
\bar{A}_{\mu}^{a}+\bar{A}_{\mu}^{a}+c^{a b c_{\theta} b_{\bar{A}_{\mu}}^{c}}-\partial_{\mu} \theta^{a},
$$

then Eq. (11) is consistent with

$$
J_{\mu}^{a}(\bar{A})+J_{\mu}^{a}+c^{a b c} \theta^{b} J_{\mu}^{c}
$$

If we make these transformations within the functional integral, Eq. (9), and if we also make the change of variables

$$
\begin{gathered}
A_{\mu}^{a}(x) \rightarrow A_{\mu}^{a}+c^{a b c_{\theta} b_{A} c} \\
\phi^{a}(x) \rightarrow \phi^{a}+c^{a b c_{\theta} b_{\phi} c} \\
\phi^{a *}(x) \rightarrow \phi^{a *}+c^{a b c_{\theta} b_{\phi} c^{\star}},
\end{gathered}
$$

then the functional integral, and hence the effective action, $\Gamma$, is shown to be invariant with respect to gauge transformations of its argument, $\overline{\mathrm{A}}_{\mu}^{\mathrm{a}}(\mathrm{x})^{12,15}$. $\Gamma(\overline{\mathrm{A}})$ does, however, depend on the choice of the background gauge parameter, $\alpha$. We will show that for the physical theory defined by Eqs. (13) 
and (14), the value of $\Gamma(\bar{A})$ is left invariant by changing $\alpha$. The value of $\bar{A}_{\mu}^{a}(J=0)$, however, is not invariant. If we keep $\overline{\mathrm{A}}_{\mu}^{\mathrm{a}}$ fixed but make the change of variables

$A_{\mu}^{a}+A_{\mu}^{a}+c^{a b c} \theta^{b}\left(A_{\mu}^{c}+\bar{A}_{\mu}^{c}\right)-\partial_{\mu} \theta^{a}=A_{\mu}^{a}-D_{\mu}^{a b}(A+\bar{A}) \theta^{b}$,

then the value of the functional integral is invariant. This change of variables is equivalent to a gauge transformation of the quantity $\left(A_{\mu}^{a}+\bar{A}_{\mu}^{a}\right)$. Therefore, the action, $\int a^{4} x L(A+\bar{A})$, is left invariant. The measure [dA] is invariant with respect to such linear transformations. The Faddeev-Popov determinant is also invariant as demonstrated below. (This follows a similar proof due to Abers and Lee $\left.{ }^{40}\right)$. Define $\Delta(A, \bar{A})$ by

$$
\Delta(A, \bar{A}) \int \delta\left[D^{\mu}(\bar{A}) A_{\mu}^{\Omega}-f(x)\right] d \Omega=1
$$

where

$$
A_{\mu}^{\Omega}=\Omega\left(A_{\mu}+\bar{A}_{\mu}\right) \Omega^{-1}+\Omega \partial_{\mu} \Omega^{-1}-\bar{A}_{\mu} .
$$

This is just the finite version of the infinitesimal transformation, Eq. (32). Also, $A_{\mu} \equiv A_{\mu}^{a_{T}}{ }^{a}$, where $T^{a}$ is a matrix representation of the group generators. Now, $A_{\mu}^{\Omega \Omega}$ ' is defined by group multiplication, i.e., $A_{\mu}^{\Omega \Omega^{\prime}}=A_{\mu}^{\Omega^{\prime \prime}}$ where 
$\Omega \Omega^{\prime}=\Omega^{\prime \prime}$. Also $d \Omega^{\prime \prime}=d\left(s i \Omega^{\prime}\right)=$ dsi. Therefore, by changiny variables $A_{\mu} \rightarrow A_{\mu} \Omega^{\prime}$, we have

$$
\Delta\left(A^{\Omega^{\prime}}, \bar{A}\right) \int \delta\left[D^{\mu}(\bar{A}) A_{\mu}^{\Omega^{\prime} \Omega}-f(x)\right] d\left(\Omega^{\prime} \Omega\right)=1 .
$$

The integral above is identical to the one in Eq. (33), since we are integrating over all $\Omega$. Therefore we must have

$$
\Delta\left(A^{\Omega^{\prime}}, \bar{A}\right)=\Delta(A, \bar{A})
$$

Eq. (33) can be solved for $\Delta(A, \bar{A})$ near the constraint surface defined by the delta function, to give

$$
\Delta(A, \bar{A})=\operatorname{det}\left[D_{\mu}^{a b}(\bar{A}) D^{\mu b c}(\bar{A}+A)\right]
$$

This is just the Faddeev-Popov determinant. Eq. (36) and (37), together, demonstrate the invariance of the FadeevPopov determinant under the change of variables, Eq. (32). The only term that we have not discussed is the gauge fixing term. In its exponentiated version, we have the exponential of the integral of $-(1 / 2 \alpha)\left[D_{\mu}^{a b}(\bar{A}) A^{\mu b}\right]^{2}$. If we make the change of variables, (32), then

$$
D_{\mu}^{a b}(\bar{A}) A^{\mu b}+D_{\mu}^{a b}(\bar{A}) A^{\mu b}-D_{\mu}^{a b}(\bar{A}) D^{\mu b c}(\bar{A}+A) \theta^{c} .
$$


If we now specify $\theta^{C}$ such that

$$
D_{\mu}^{a b}(\bar{A}) D^{\mu b c}(\bar{A}+A) \theta^{c}=\frac{\delta \alpha}{2 \alpha} D_{\mu}^{a b}(\bar{A}) A^{\mu b}
$$

where $\delta \alpha$ is an infinitesimal, then

$$
\begin{aligned}
-\frac{1}{2 \alpha}\left[D_{\mu}^{a b}(\overline{\mathrm{A}}) A^{\mu b}\right]^{2} & --\frac{1}{2 \alpha}\left(1-\frac{\delta \alpha}{2 \alpha}\right)^{2}\left[D_{\mu}^{a b}(\bar{A}) A^{\mu b}\right]^{2} \\
& \simeq-\frac{1}{2(\alpha+\delta \alpha)}\left[D_{\mu}^{a b}(\bar{A}) A\right]^{\mu b} .
\end{aligned}
$$

Therefore, the only effect of changing variables with the transformation (32) and (39) is to change the value of $\alpha$ to $\alpha+\delta \alpha$. Since the functional integral is invariant under changes of integration variables, it is therefore independent of the choice of $\alpha$. (If $J_{\mu}^{a} \neq 0$, this is no longer true.) Furthermore, 't Hooft demonstrates that apart from renormalizations due to fermions or scalar fields in the theory, the infinities of $\Gamma$ will be gauge independent. Thus we can construct a gauge invariant renormalization scheme for the vector part of the theory. When $J_{\mu}^{a}(\bar{A})=0$, the one loop approximation to the above formalism is equivalent to one in which $\bar{A}_{\mu}^{a}(x)$ satisfies the classical equations of motion. The oneloop diagrams satisfy the ordinary ward identities rather than the slavnov-Lee identities, and the only renomalization needed is a coupling constant renomalization. $12,14,15$ 
III. THE CONSTANT MAGNETIC FIELD AND ZERO POINT ENERGY

The calculation of the one-loop approximation to the effective action as the sum over the zero point energies of the theory has been suggested in the work of Nielsen and olesen ${ }^{16}$ where they consider the one-loop effective action in the presence of a constant color magnetic field. They advocate the study of such field configurations as a tool in understanding quark confinement. Although vacuum configurations in which $\langle\overrightarrow{\mathrm{H}}\rangle \neq 0$ are not Lorentz invariant or even rotationally invariant, Nielsen and Olesen suggest that such configurations are important, for the apparent instabilities in these configurations may indicate something about the true nature of the Yang-Mills vacuum. Along this line, Ambjorn, Nielsen and olesen have suggested a theory based upon a condensate of color magnetic flux tubes.7,27-31 Mandelstam ${ }^{42}$ has suggested that the instabilities for constant $H$ indicate an enhancement of the virtual, low frequency modes in a Fourier expansion of the magnetic field. ' $t$ Hooft ${ }^{43}$ has indicated how to study the local stability of theories with non-zero, topologically stable magnetic flux. A justification for these programs lies in the result that the real part of the energy is lowered by the oneloop term in the presence of a constant magnetic field and in the interpretation of the calculated imaginary part of the effective action as an indication of an instability leading to a lower energy, stable ground state. 
The first indication that the real part of the energy is lowered for small external color magnetic fields was implicit in the work of Vanyashin and Terent'ev ${ }^{44}$ where they noted the "anomalous character of the charge renormalization" for a charged vector field in the presence of an external magnetic field. A decade later, Duff et al. 23 used schwinger's elegant proper time technique to write down the effective Lagrangian for a non-abelian theory in the presence of general external fields. The first systematic calculation of the explicit one-loop effective action for a constant external magnetic field using both proper time and renormalization group techniques was by savvidy, Matinyan et al.19-2l However, they failed to note the imaginary part. Nielsen and olesen ${ }^{16}$ redid the calculation by assuming that the one-loop term can be written as the change in the zero point energy of the theory. Falomir and Schaposnik ${ }^{18}$ extended the calculation to also include scalar particles in the adjoint representation, but their calculation appears to depend on their choice of gauge. Yildiz and $\operatorname{cox}^{22}$ also perform the calculation, in this case including scalars and spinors in an arbitrary representation, using the definition of the effective action due to schwinger. Flyvbjerg 16 has also redone the calculation for the pure vector theory with gauge groups su(3) and SU(4), emphasizing novel renormalization effects not found in SU(2). The last four papers all use proper time techniques to evaluate their integrals, and all note the existence of an imaginary part. 
The one-loop approximation to a pure vector gluon theory gives the following for the real part of the effective energy density ${ }^{17}$

$$
\operatorname{Re} E=\frac{1}{2} \mathrm{H}^{2}+\frac{11 \mathrm{~N}}{96 \pi^{2}} g^{2} \mathrm{H}^{2}\left(\ln \frac{g \mathrm{H}}{\Lambda^{2}}-\frac{1}{2}\right)
$$

where $\mathrm{N}$ depends on the gauge group $\mathrm{SU}(\mathrm{N})$. Renormalization group arguments can be given to show that this is a good approximation for large $H, i . e .$, for $g H \gg \Lambda^{2}$ where $\Lambda^{2}$ is the renormalization point.16-22 If the above approximation is extended to smaller values of $\mathrm{H}$, it is noted that there exists a minimum away from $H=0$. For the above approximation to hold in this region it is required that

$$
\int_{g}^{\infty} \frac{d g}{B(g)}<\infty
$$

where $B(g)$ is the usual Callan-symansik beta function. This behavior of $B(g)$ corresponds to the "ferromagnetic" classification of the theory due to Pagels and Tomboulis 45 and Gross and wilczek. 46 For this to happen, the running coupling constant $\bar{g}^{2}(\Lambda)$ must become negative, a pathological result, unless a phase transition to some ordered system occurs. This is just what Nielsen and olesen suggest, although the proof must somehow come from the theory itself. 
As already stated, Nielsen and Olesen suggest that the one-loop approximation to the effective action can be written as the difference in the zero point energy of the theory due to the presence of an external vector field. This result has previously been found for particle theories by coleman ${ }^{l}$ and for scalar field theories by salam and Strathdee. 24 It was also pointed out by Dolan and Jackiw 26 and by weinberg, 25 as the zero temperature limit of scalar and fermion field theories formulated at finite temperature. The extension to vector gauge theories is complicated by the gauge problems associated with the construction of the effective action. The zero point energy of the theory is a gauge invariant quantity, and hence problems with the gauge non-invariance of the effective action would naturally lead to problems with this interpretation. With the formulation of the background field method of section 2 , the effective action is gauge invariant and we are able to show the equivalence of the one-loop term to the change in the zero-point energy due to the presence of an external field. We will now demonstrate this result for a limited class of external background fields, $\bar{A}_{\mu}^{a}(x)$, and clarify to what extent this class may be enlarged without changing the interpretation. We will then verify the result explicitly for the case of a constant external magnetic field. 
To calculate the one-loop approximation for $\Gamma$, we expand the exponential in Eq. (9) in powers of $A_{\mu}$. Since we are interested in vacuum configurations, we take the case $J_{\mu}^{a}(\bar{A})=0$. This is therefore the gauge invariant formulation of the theory. The one-loop approximation is found by keeping only quadratic terms in the fields $A_{\mu}^{a}$, $\phi^{a}$ and $\phi^{a^{*}}$. The effective action to this order is then

$$
\begin{aligned}
& \Gamma(\bar{A})=\int d^{4} x L(\bar{A})-i \hbar \ln N \int[d A][d \dot{\phi}]\left[d \phi^{*}\right] x \\
& x \exp -\frac{i}{2 \hbar} \int d^{4} x\left[\left[D_{\mu}(\bar{A}) A_{\nu}\right]^{a}\left[D^{\mu}(\bar{A}) A^{\nu}\right]^{a}-\left[D_{\mu}^{-}, \bar{T}\right) A^{\mu b}\right]^{2}+ \\
& \left.+2 g F^{\mu \nu a}(\bar{A}) c^{a b c} A_{\mu}^{b}{ }_{\nu}+\frac{1}{\alpha}\left[D_{\mu}^{a b}(\bar{A}) A^{\mu b}\right\}^{2}\right\} \\
& \left.+2 \phi^{a *}\left[D_{\mu}(\bar{A}) D^{\mu}(\bar{A})\right]^{a b} \phi^{b}\right\}
\end{aligned}
$$

where

$$
\begin{gathered}
L(\bar{A})=-\frac{1}{4} F_{\mu \nu}^{a} F^{\mu \nu a} \\
F_{\mu \nu}^{a}(\bar{A})=\partial_{\mu} \bar{A}_{\nu}^{a}-\partial_{\nu} \bar{A}_{\mu}^{a}+g c^{a b c_{\bar{A}}} b_{\mu} \bar{A}_{\nu}^{c}
\end{gathered}
$$

and the normalization $\mathrm{N}$ is defined so that the one-loop term (the logarithm above), $\Gamma_{1}$, vanishes when $\bar{A}_{\mu}^{a}=0$. Note also that: 


$$
B^{i a}=-\frac{1}{2} \varepsilon^{i j k_{F}^{a}}{ }_{j k}
$$

and

$$
E^{i a}=F^{i o a}
$$

As stated in Section 2, to the one-loop order we may freely choose $\overline{\mathbb{A}}_{\mu}^{a}$ to be solutions of the classical equations of motion. Integrating by parts, we find that the one-loop term alone becomes:

$$
\begin{aligned}
\Gamma_{I}(\bar{A})= & -i \hbar \ln N \int[d A][d \phi]\left[d \phi^{*}\right] \times \\
& \times \exp \frac{i}{2 \hbar} \int a^{4} x\left\{A ^ { \mu a } \left[g_{\mu \nu}\left(D_{\alpha}(\bar{A}) D^{\alpha}(\bar{A})\right) a c\right.\right. \\
& \left.+2 g c^{a b c} F_{\mu \nu}^{b}(\bar{A})\right] A^{\nu C}- \\
& -\phi^{a *}\left[D_{\alpha}(\bar{A}) D^{\alpha}(\bar{A})\right]^{a c c_{\phi} c}
\end{aligned}
$$

where we have used our gauge freedom to put $\alpha=1$. To evaluate the functional integral, we will define the theory in Euclidean space by first letting

$$
\begin{gathered}
t \rightarrow-i \tau \\
A^{\circ}+-i A^{4} .
\end{gathered}
$$


The Euclidean theory is defined from this change of variables by letting the integral over $\tau$ go from $-\infty$ to $+\infty$. Then

$$
z_{E}\left(\bar{A}_{E}\right) \equiv \exp \left[-\frac{1}{\hbar} \Gamma_{E}\left(\bar{A}_{E}\right)\right]
$$

This implies that

$$
\Gamma=\left.i \Gamma_{E}\right|_{\substack{\tau=i t \\ A^{4}=i A^{\circ}}}
$$

Henceforth, all subscripts $E$ will be dropped, and all four vectors are Euclidean unless otherwise noted. The Euclidean one-loop term becomes:

$$
\begin{aligned}
& \Gamma_{I}(\bar{A})=-\hbar \ln N \int[d A][d \phi]\left[d \phi{ }^{*}\right] x \\
& x \exp -\frac{1}{2 \bar{H}} \int a^{4} \times\left\{A ^ { \mu b } \left[\delta_{\mu \nu}\left(D_{\alpha}(\bar{A}) D^{\alpha}(\bar{A})\right)^{b c}\right.\right. \\
& \left.-2 g c^{a b c_{F} a}(\bar{A})\right] A^{\nu c}+ \\
& \left.+2 \phi^{a *}\left(D_{\alpha}(\bar{A}) D^{\alpha}(\bar{A})\right)^{a b_{\phi} b}\right\}
\end{aligned}
$$

where now the metric is $-\delta_{\mu \nu}$.

We shall label the bracketed differential operator in Eq. $(50), G_{\mu \nu}^{b c}(x)$. The functional integral over $A_{\mu}^{a}$ is then formally equal to 


$$
\operatorname{det}\left[G_{\mu \nu}^{b c}(x) \delta^{4}(x-y)\right]^{-\frac{1}{2}}
$$

where we have assumed that the eigenvalues of $G_{\mu \nu}^{b c}$ are positive definite in order for the Gaussian integral to be well defined. Rather than proceeding as above, we can examine the integral mode by mode by expanding the fields $A^{\mu a}(x)$ in a basis which diagonalizes $G_{\mu \nu}^{a b}$. since $G_{\mu \nu}^{a b}$ is a differential operator, it is not diagonal in coordinate space. As we shall see, in order to show the equivalence of $\Gamma_{1}$ to a sum over zero point energies, we need $G_{\mu \nu}^{a b}$ to be diagonal in frequency space. To effect this we shall restrict $\overline{\mathrm{A}}_{\mu}^{\mathrm{a}}(\mathrm{x})$ and later we will examine to what extent this restriction can be relaxed without affecting the result. Since $\Gamma(\bar{A})$ is gauge invariant we will use this freedom to put $\overline{\mathrm{A}}_{\mu}^{a}$ in the temporal gauge, $\bar{A}_{4}^{a}=0$. (This still leaves us the freedom to make an additional time independent gauge transformation.) Now we will restrict $\bar{A}_{i}^{a}(x)$ to be static. This is a genuine constraint, but it is not very severe since our purpose is to find field configurations of lowest energy and in many cases this can be assumed to be static. (Jackiw and Rossi 47 have demonstrated the existence of time dependent classical solutions which give rise to lower energies than certain "gyroscopically" stable static configurations in the classical theory with sources. 
It is still presumed, however, even in that theory, that the lowest energy field configurations would still be static. This has been shown in the work of Sikivie and Weiss 48 and Magg. ${ }^{49}$, with this restriction $G_{\mu \nu}^{b c}$ takes the form:

$$
G_{\mu \nu}^{b c}=-\delta_{\mu \nu} \delta^{b c} \partial_{4}^{2}+M_{\mu \nu}^{b c}(\vec{x})
$$

Now by a similarity transformation let us diagonalize $G_{\mu \nu}^{b c}$ in Lorentz space and group space. This is a bit tricky (since $G$ is also a differential operator) and has only been done for a countable set of external field configurations, among which are constant fields and plane waves. 50 Assuming that $G$ is still in the form of Eq. (52), we now expand $A_{\mu}^{a}(x)$ in normalized eigenfunctions $x_{n}(\vec{x})$ of the differential operator $G$ :

$$
A_{\mu}^{a}=\sum_{n} \int \frac{d k_{4}}{\sqrt{2 \pi}} c_{\mu, n}^{a}\left(k_{4}\right) e^{i k_{4}^{\tau}} x_{n}(\vec{x})
$$

where $n$ may be either a discrete or continuous variable (or both). Then

$$
G_{\mu \nu}^{b c_{A} \nu c}=\sum_{n} \int \frac{a k_{4}}{\sqrt{2 \pi}}\left[\delta_{\mu \nu} \delta b c_{k_{4}^{2}}+\lambda_{n, \mu \nu}^{b c}\right] c_{n}^{\nu c} e^{i k_{4}^{\tau}} x_{n}(\vec{x})
$$

where $\lambda$ is diagonal in Lorentz and group indices. Then the integral over $A_{\mu}^{a}$ in Eq. (50) becomes 


$$
\begin{aligned}
& N^{\prime} \int[a c] \exp -\frac{1}{2 \pi} \sum_{n, m} c_{m}^{\mu b}\left(k_{4}\right)\left(s_{\mu \nu} s^{b c k_{4}^{2}}+\lambda_{n, \mu \nu}^{b c}\right] s_{m} s\left(k_{4}-k_{4}^{\prime}\right) c_{n}^{k c}\left(k_{4}^{\prime}\right) \\
& =N^{\prime} \operatorname{det}\left[\left(\delta_{\mu \nu} \delta b k_{4}^{2}+\lambda_{n, \mu \nu}^{b c}\right) \delta_{m n} \delta\left(k_{4}-k_{4}^{\prime}\right)\right]^{-\frac{1}{5}}
\end{aligned}
$$

where $N$ ' has been defined to include any field independent constant in the transformation from $A_{\mu}^{a}(x) \rightarrow c_{\mu, \Omega}^{b}\left(k_{4}\right)$. Exponentiating the determinant, we find that Eq. (50) becomes

$$
\begin{aligned}
\Gamma_{1}(\bar{A})= & N \nVdash \operatorname{Tr} \delta_{m n} \delta\left(k_{4}-k_{4}^{\prime}\right)\left[\frac{1}{2} \ln \left(\delta_{\mu \nu} \delta c_{k_{4}}^{2}+\lambda_{n, \mu \nu}^{b c}\right)-\right. \\
& \left.-\ln \left(\delta_{\mu \nu} \delta b c_{4}^{2}+\Lambda_{n, \mu \nu}^{b c}\right)\right]
\end{aligned}
$$

where the second term in the brackets comes from the ghost part of the exponential and is of opposite sign (or power) because of the antisymmetry of the $\phi^{a}$ fields. The extra factor of two arises from an integration over both the real and imaginary parts of $\phi^{a}$. The determinant in Eq. (55) has as its argument terms proportional to the unit operator $\delta_{\mathrm{mn}} \delta\left(k_{4}-k_{4}^{\prime}\right)$, which have been slipped through the logarithms above. Now taking the trace, we set $m=n, \mu=v$, $b=c$, let $k_{4}^{\prime} \rightarrow k_{4}$ and sum over these diagonal elements. Let us now do the integral over $k_{4}$ for one of these terms. Following the procedure in Salam and strathdee, ${ }^{24}$ we let 


$$
\begin{aligned}
N \int d k_{4} \ln \left(k_{4}^{2}+\lambda_{n}\right)= \\
=\iint_{\lambda_{n}(0)}^{\lambda_{n}(\bar{A})} d \lambda_{n}^{\prime} \int d k_{4} \frac{d}{d \lambda_{n}^{\prime}} \ln \left(k_{4}^{2}+\lambda_{n}^{\prime}\right)
\end{aligned}
$$

where the limits of integration have been set to absorb the normalization $N$, i.e., $N$ is defined so that $\lim _{\bar{A} \rightarrow 0} \Gamma_{I}(\bar{A})$ $=0$. Performing the differentiation, we have

$$
\int_{\lambda_{n}(0)}^{\lambda_{n}(\bar{A})} d \lambda_{n}^{\prime} \int_{-\infty}^{+\infty} \frac{d k_{4}}{k_{4}^{2}+\lambda_{n}^{\prime}} \text {. }
$$

In assuming that the eigenvalues of $G$ are positive definite, we note that $\lambda_{n}^{\prime}$ must also be positive definite. (When $k_{4}=0$, the eigenvalues of $G$ are equal to $\lambda_{.}$) In this case, the above integral reduces to

$$
\begin{gathered}
\left.\int_{\lambda_{n}(0)}^{\lambda_{n}(\bar{A})} d \lambda_{n}^{\prime} \frac{1}{\sqrt{\lambda_{n}^{\prime}}} \operatorname{Tan}^{-1}\left(\frac{k_{4}}{\lambda_{n}^{\prime}}\right)\right|_{-\infty} ^{+\infty}= \\
=2 \pi\left[\sqrt{\lambda_{n}(\bar{A})}-\sqrt{\lambda_{n}(0)}\right] .
\end{gathered}
$$

Therefore, Eq. (56) reduces to

$\Gamma_{I}(\bar{A})=\int \mathrm{d} \tau \delta_{\mathrm{mm}} \sum_{n, \mu, b} \frac{\hbar}{2}\left\{\left[\sqrt{\lambda_{n, \mu}^{b}(\bar{A})}-\sqrt{\lambda_{n, \mu}^{b}(0)}\right]-2\left[\sqrt{\Lambda_{n, \mu}^{b}(\bar{A})}-\sqrt{\Lambda_{n, \mu}^{b}(0)}\right]\right\}$

where a single index on $\lambda$ implies a diagonal element and where 


$$
\frac{1}{2 \pi} \int d \tau=\lim _{k_{4}^{\prime} \rightarrow k_{4}} \frac{1}{2 \pi} \int d \tau e^{i\left(k_{4}^{\prime}-k_{4}\right) \tau}=\lim _{k_{4}^{\prime} \rightarrow k_{4}} \delta\left(k_{4}^{\prime}-k_{4}\right) \text {. }
$$

Now writing $\Gamma_{I}(\bar{A})=\int d \tau I_{I}(\bar{A})$, we note that the first term in $L_{1}$ looks like the change in the zero point energy of the theory due to the presence of the external field $\bar{A}$. To see that $\sqrt{\lambda}$ is an energy, continue the operator $G_{\mu \nu}^{b c}(\bar{A})$ back to Minkowski space where it is equivalent to the inverse propagator. If we examine its decomposition into normal modes, Eq. (56), we note that in Minkowski space the zeroes of the energy transform (poles in the propagator) occur for $k_{0}^{2}=\lambda$. Therefore, the $\sqrt{\lambda(\bar{A})}$ are just vector particle energy eigenvalues.

The second term in Eq. (60) is the contribution from the ghost determinant. It is of the same form as the first term and will be seen in specific examples to cancel the contribution from any unphysical degrees of freedom.

Continuing back to Minkowski space via Eq. (49), we now get

$$
\begin{aligned}
\Gamma_{I}(\bar{A})= & -\int \operatorname{dt} \delta_{m m} \sum_{n, \mu, b} \frac{\hbar}{2}\left\{\left[\sqrt{\lambda_{n, \mu}^{b}(\bar{A})}-\sqrt{\lambda_{n, \mu}^{b}(0)}\right]-\right. \\
& \left.-2\left[\sqrt{\Lambda_{n, \mu}^{b}(\bar{A})}-\sqrt{\Lambda_{n, \mu}^{b}(0)}\right]\right\} .
\end{aligned}
$$

Therefore, the one-loop correction to the effective Lagrangian is the negative of the change in zero point energy in the 
presence of an external field $\bar{A}_{\mu}^{a}$, where $A_{0}^{a}=0$ and $A_{i}^{a}(x)$ $=A_{i}^{a}(\vec{x})$. For this field configuration the electric field $E_{i}^{a}(x)=0$, and thus the kinetic energy is zero. Therefore, the Lagrangian is minus the potential energy, so that the one-loop contribution to the energy is just the change in zero point energy. This agrees with our intuition, since in the presence of an external field, the zero point energy has changed. We have, naively, normalized the energy for $\bar{A}_{\mu}^{a}=0$ by neglecting the zero point energy (normal ordering). In the presence of an external field, the zero point energy is now changed, and since zero point energy is a quantum phenomena, this change should appear in the first quantum correction to the energy. Another way of stating the same thing is that due to the initial $(\bar{A}=0)$ normal ordering of the theory, the change in zero point energy shows up at the one loop order as zero point fluctuations around the original zero point energy. This is analogous to the argument given by coleman 41 of a particle sitting at the minimum of a potential. To get the first quantum correction to the energy, we approximate the potential by a harmonic oscillator and add the zero point energy of the oscillator. This interpretation is also similar to one implicit in the work of Weinberg ${ }^{25}$ in calculating higher order corrections to theories at finite temperatures. 
We may now ask what happens for $E_{i}^{a} \neq 0$. For time dependent configurations which are not gauge equivalent to the previous case, our simple result clearly does not follow. We cannot separate out the $\mathrm{k}_{4}$ dependence as needed. These configurations correspond to excited states of our system and the generalization of the discussion in the last paragraph would thus be more complicated. See Ref. 51 for an interpretation of $\Gamma$ for time dependent external field configurations. For configurations in which $\overline{\mathrm{A}}_{4}^{\mathrm{a}} \neq 0$, but $\overline{\mathrm{A}}_{\mu}^{\mathrm{a}}$ is still static, there would only be an addition to Eq. (54) linear in $\mathrm{k}_{4}$. In this case it might also be possible to get a zero. point energy interpretation if one could complete the square and change variables to regain a formula similar to Eq. (54). Then the Euclidean one-loop effective action would resemble Eq. (60) with appropriate $\overline{\mathrm{A}}_{4}^{\mathrm{a}}$ dependence. This linear term in $k_{4}$ is similar in structure to a gyroscopic term in the sense of Ref. 47. For this situation the transition from $\Gamma_{E}$ to $\Gamma$ is not as straightforward since we must consider the $\bar{A}_{4}^{a}$ for $\bar{A}_{0}^{a}$ ) dependence. Also since $E_{i}^{a} \neq 0$, the Lagrangian is not equal to minus the energy. However, if we define an effective energy density equal to the Euclidean Lagrangian, we would still be able to use our zero point energy interpretation for such "gyroscopic" configurations.

When $\bar{A}_{\mu}^{a}(x)$ represents a constant color magnetic field we may take

$$
\overline{\mathrm{A}}_{\mu}^{\mathrm{a}}(\mathrm{x})=\delta^{\mathrm{a} 3 \overline{\mathrm{A}}_{\mu}}(\mathrm{x})
$$


i.e., $\overline{\mathrm{A}}_{\mu}^{a}(\mathrm{x})$ points in the third direction in color space. (Here we are taking $A_{\mu}^{a}$ to be in the adjoint representation of the gauge group $\mathrm{SU}(2)$. ) Also let

$$
\begin{gathered}
\overline{\mathrm{A}}^{0}=\overline{\mathrm{A}}^{\mathrm{Y}}=\overline{\mathrm{A}}^{z}=0 \\
\overline{\mathrm{A}}^{\mathrm{X}}=-\mathrm{BY}
\end{gathered}
$$

so that from Eq. (45)

$$
B^{i a}=\delta^{i 3} \delta^{a} B
$$

i.e., $B^{i a}$ points in the third direction in both space and color space. Then define

$$
\begin{aligned}
& T^{b c} \equiv\left(\begin{array}{lll}
1 & & \\
& 0 & \\
& & -1
\end{array}\right) \\
& S_{\mu \nu} \equiv\left(\begin{array}{llll}
0 & & & \\
& 1 & 0 & \\
& & 0 & -1
\end{array}\right)
\end{aligned}
$$

and Eq. (56) becomes (again in Euclidean space)

$$
\begin{aligned}
\Gamma_{I}(\bar{A}) & =N K \operatorname{Tr} \delta\left(k_{4}^{\prime}-k_{4}\right) \delta\left(k_{x}^{\prime}-k_{x}\right) \delta\left(k_{z}^{\prime}-k_{z}\right) \delta_{m n} \times \\
& \times\left\{\frac { 1 } { 2 } \operatorname { l n } \left(\delta_{\mu \nu}\left[\delta^{b c}\left(k_{4}^{2}+k_{z}^{2}\right)+(2 n+1) g\left(T^{2}\right) b c_{B}\right]+2 g S_{\mu \nu} T^{\left.b c_{B}\right)}\right.\right. \\
& \left.-\ln \delta_{\mu \nu}\left[\delta^{b c}\left(k_{4}^{2}+k_{z}^{2}\right)+(2 n+1) g\left(T^{2}\right) b c_{B}\right]\right\} .
\end{aligned}
$$


Upon taking the trace, we find that the ghost term cancels with the two terms corresponding to the zero eigenvalues of $s_{\mu \nu}$, leaving

$$
\begin{aligned}
\Gamma_{I}(\bar{A})= & N \frac{\pi}{2} \int d \tau d x d z \sum_{n} \int \frac{d k_{4} d k_{z} d k_{x}}{(2 \pi)^{3}} x \\
& \times 2\left\{\ln \left[k_{4}^{2}+k_{z}^{2}+(2 n+3) g B\right]\right. \\
& \left.+\ln \left[k_{4}^{2}+k_{z}^{2}+(2 n-1) g B\right]\right\}
\end{aligned}
$$

Notice the integral over $d k_{x}$. Since the integrand is independent of $k_{x}$, this is analogous to a zero eigenvalue problem. This reflects a symmetry of the theory that we have overlooked. Instead of integrating over $\mathrm{k}_{\mathrm{x}}$ we can put our problem in a box. 52 The sum over $n$ above comes from the sum over the energy eigenvalues of a linear harmonic oscillator in the $y$ direction of the form

$$
p_{y}^{2}+(g B)^{2}\left(y \mp y_{0}\right)^{2}
$$

where

$$
y_{0}=P_{x} / g B
$$

Therefore, in integrating over $k_{x}$, we are summing over 
all possible locations of the origin of the oscillator. If we put our system in a box of length $I_{y}$, then $0<y_{0}<I_{y}$ or $0<\mathrm{P}_{\mathrm{x}}<\mathrm{gBI}_{\mathrm{y}}$. Therefore, the integral over $\mathrm{k}_{\mathrm{x}}$ should just give $g B L_{y}$. If we write $I_{y}$ as $\int d y$, then

$$
\begin{aligned}
\Gamma_{1}(\bar{A})= & N \frac{\hbar}{2} \frac{g B}{2 \pi} \int d^{4} x \sum_{n} \frac{d k_{4}}{2 \pi} \frac{d k_{z}}{2 \pi} \times \\
& \times 2\left\{\ln \left[k_{4}^{2}+k_{z}^{2}+(2 n+3) g B\right]\right. \\
& \left.+\ln \left[k_{4}^{2}+k_{z}^{2}+(2 n-1) g B\right]\right\} .
\end{aligned}
$$

Now note that, for $n=0$ and $k_{4}^{2}+k_{z}^{2}<g B$, the second logarithm has a negative argument. This traces back to the existence of negative eigenvalues for the operator $\mathrm{G}_{\mu \nu^{\prime}}^{\mathrm{bc}}$ and hence the Euclidean formulation of the theory is ill-defined. We will, for the moment, naively proceed with our analysis, overlooking this problem, but we will return to it in the next section. If we do so, then in analogy to Eq. (62) we would get in Minkowski space:

$$
\begin{aligned}
\Gamma_{1}(\bar{A})= & -\int d^{4} x \sum_{n} \frac{g B}{2 \pi} \int \frac{d k z}{2 \pi} \times \\
& \times\left\{\frac { \hbar } { 2 } \left[2 \sqrt{k_{z}^{2}+(2 n+3) g B}+2 \sqrt{\left.k_{z}^{2}+(2 n-1) g B\right]}\right.\right. \\
& \left.-\frac{\hbar}{2}\left[2 \sqrt{k_{z}^{2}}+2 \sqrt{k_{z}^{2}}\right]\right\} .
\end{aligned}
$$


Therefore, we have our result for constant magnetic field $B$. We can easily see the source of the imaginary part of $r$. For $\mathrm{n}=0$ and $\mathrm{k}_{\mathrm{z}}^{2}<\mathrm{gB}$, the second term above will be imaginary. Thus by writing the effective action as a sum over zero point energies, we can readily distinguish the real and the imaginary parts. We should note, however, that some of the steps used in the above derivation are in fact ill-defined for these imaginary energy field modes. In the next section we will examine these field modes carefully and give an indication of when the one-loop approximation is still sensible. We will also investigate the interpretation of the imaginary part of the effective action and we will indicate the circumstances for which it may be useful in discussing local stability. 


\section{NEGATIVE EIGENVALUES AND INSTABIIITY}

The imaginary part of the one-loop term of the effective action in the presence of a constant color magnetic field was first noted by Nielsen and olesen 16 as due to the anomalous magnetic moment of the gluon $\left(2 g c^{a b c_{F} b}(\bar{A})\right.$ in Eq. (44)). The result was implicit, however, in earlier works, ${ }^{32}$ where the energy eigenvalues for vector fields with anomalous magnetic moments and electric quadrupole moments were determined. This work was then specialized to non-abelian gauge theory and tachyonic states were noted. 32 An instability in the presence of a constant color magnetic field has also been pointed out in the classical theory by Chang, Weiss and Sikivie, 33,34 and more recently by Cosenza and Neri. 35 The precise relationship between the classical instability and the imaginary part of the effective action at the oneloop (semi-classical) level has not been pointed out. In this section, we shall investigate the negative eigenvalue modes and in doing so will show that a relationship does indeed exist between the classical and semi-classical instabilities. We shall evaluate the validity of the oneloop approximation in the presence of negative eigenvalues and demonstrate how to "regularize" the functional integral in order to calculate the imaginary part. In this context, we can then give a physical interpretation of the imaginary part and connect this interpretation to that of imaginary 
effective potentials in the context of nucleation ${ }^{38}$ or the "decay of the false vacuum"36,37 and pair production in constant electric fields. 6

As noted after Eq. (71), there exists negative eigenvalues for the operator $G_{\mu \nu}^{b c}$ in the presence of a constant color magnetic field. It can be shown that these are just due to the anomalous magnetic moment of the gluon, as noted above. The Euclidean functional integral, Eq. (55), for these modes will look, mode by mode, like

$$
\int_{-\infty}^{+\infty} d C \exp +\frac{1}{2} C\left(g B-k_{4}^{2}-k_{z}^{2}\right) C \rightarrow \infty
$$

where we have suppressed indices. These are just the modes for which, in the second term of Eq. (68), $\mathrm{n}=0$. For $k_{4}^{2}+k_{z}^{2}<g B$, the integral above is clearly infinite, and thus the Euclidean functional integral diverges. This does not mean that the theory is ill-defined since we are only looking at the loop approximation. It does appear, however, that the loop approximation is invalid in the case of a constant color magnetic field. Let us be more careful and see. if there is any information that we may salvage. Imagine a theory such that

$$
z=\pi_{k} \int d \phi_{k} \exp -\frac{1}{2} \Delta_{k}\left(k^{2} \phi_{k}^{2}-m^{2} \phi_{k}^{2}+\lambda \phi_{k}^{4}\right) .
$$


If this integral is expanded around $\phi=\phi_{0}=0$, then to quadratic order, when $\mathrm{m}^{2}>\mathrm{k}^{2}$, we will have the same form as Eq. (73), i.e., a tachyon (negative (mass) ${ }^{2}$ ) is present. Here we are expanding around a maximum rather than a minimum of the potential (see Fig. 1). Clearly, a better approximation is to expand around one of the two symmetric minima. For the gauge theory problem, we cannot solve for the minima, but the existence of negative eigenvalues indicates that we are expanding about a "bad" field configuration. Note that an expansion about $\phi=0$ in Fig. 1 is also classically unstable.

In a classical analysis, we demand for stability that we expand around a field configuration which minimizes the energy, unless, as shown by Jackiw and Rossi, ${ }^{47}$ there exist gyroscopic terms in the Hamiltonian. (For a review of gyroscopic stability and an extension of the following analysis to cases where gyroscopic terms are present, see Appendix B.) Let

$$
H=\frac{1}{2} \pi^{2}+V(\phi)+G \pi \phi
$$

For the case $G=0$ (no gyroscopic terms), if we expand $H$ around static solutions of Hamilton's equations:

$$
\dot{\phi}_{C l}=\left.\frac{\delta H}{\delta \pi}\right|_{\pi_{C l}}=0, \dot{\pi}_{C l}=-\left.\frac{\delta H}{\delta \phi}\right|_{\phi_{C l}}=-\left.\frac{\delta V}{\delta \phi}\right|_{\phi C l}=0
$$

then

$$
\frac{\delta^{2} H}{\delta \pi^{2}}=1,\left.\frac{\delta^{2} H}{\delta \phi^{2}}\right|_{\phi c l}=\left.\frac{\delta^{2} V}{\delta \phi^{2}}\right|_{\phi_{C l}} .
$$


For minimality we require that $\left.\left(\delta^{2} \mathrm{v} / \delta \phi^{2}\right)\right|_{\phi_{c l}}>0$.

Now, in the Euclidean formulation of the functional integral, the argument of the exponential, modulo gauge fixing terms and ghosts, is equal to minus the Euclidean action. The general structure of the Lagrangian density is

$$
L(\phi)=\frac{1}{2}\left(\frac{\partial \phi}{\partial t}\right)^{2}+G \phi \frac{\partial \phi}{\partial t}-\tilde{V}(\phi) .
$$

If we again specialize to the case $G=0$, then

$$
\hat{v}(\phi)=v(\phi) \text {. }
$$

To go to the Euclidean formulation let

$$
L_{E}=-\left.L\right|_{t \rightarrow-i \tau}
$$

or

$$
L_{E}(\phi)=\frac{1}{2}\left(\frac{\partial \phi}{\partial \tau}\right)^{2}+V(\phi)
$$

To calculate the one-loop term we expand $L_{E}$ around static solutions of the classical Euclidean equations of motion:

$$
\left.\frac{\partial^{2} \phi}{\partial \tau^{2}}\right|_{\Phi_{E}}=\left.\frac{\delta V}{\delta \phi}\right|_{\Phi_{E}}=0,
$$


and the quadratic term becomes

$$
-\left.\frac{1}{2} \phi \frac{\delta^{2} L_{E}}{\delta \phi^{2}}\right|_{\phi_{E}} \phi
$$

Therefore, for the theory to be well defined, we demand positivity of the eigenvalues of the above kernel, i.e.

$$
\left.\frac{\delta^{2} L_{E}}{\delta \phi^{2}}\right|_{\Phi_{E}}>0
$$

Explicitly:

$$
\left.\frac{\delta^{2} L_{E}}{\delta \phi^{2}}\right|_{\Phi_{E}}=-\frac{\partial^{2}}{\partial \tau^{2}}+\left.\frac{\delta^{2} v}{\delta \phi^{2}}\right|_{\Phi_{E}}
$$

Now, under the assumption that we may separately diagonalize the two terms above, the first term will always be positive semi-definite. Now from Eq. (80) and Eq. (76), $\phi_{c l}=\Phi_{E}$. If our theory is classicallystable, $\left.\left(\delta^{2} v / \delta \phi^{2}\right)\right|_{\phi_{C l}}>0$, then it will also give rise to positive eigenvalues at the oneloop level. Also, if we have negative eigenvalues, $\left.\left(\delta^{2} \mathrm{~V} / \delta \phi^{2}\right)\right|_{\Phi_{E}}<0$, then the theory must be classically unstable. Note that it is possible to be classically unstable and still have positive eigenvalues.

There are two apparent exceptions to these conclusions. The first occurs in the theory of metastability due to 
Lange ${ }^{38}$ and Callan and Coleman. ${ }^{36}$ In this case we may expand the theory locally around a minimum of the potential, but quantum tunneling renders the theory unstable. However, the quantum tunneling amplitude is a non-perturbative, Euclidean time dependent effect while our analysis is for static, perturbative fields.

The second exception occurs in the work of coleman and Weinberg 53 when considering theories with two coupling consstants. In this case the one-loop effects due to one coupling may be of the same magnitude as the tree terms due to the other coupling. Thus, a minimum of the classical potential may turn into a local maximum when one-loop effects are included. In this case, then, there is a grey area in the distinction between classical and semiclassical approximations, for which we may consider an effective classical theory which does not encounter this problem.

For gauge theories things are not quite so simple. To go to Euclidean space $t \rightarrow-i \tau$ and $A_{0}^{a} \rightarrow-i A_{4}^{a}$. It can be shown, however (see Appendix A), that the above analysis does follow for the case in which we expand about static solutions for which $\overline{\mathrm{A}}_{4}^{a}=0$. If $\overline{\mathrm{A}}_{4}^{\mathrm{a}} \neq 0$, then there are gyroscopic terms (see Appendix B.4) and classical stability does not imply 
minimality. 47 It is probable, however, that a comparison can be made between positivity of the real part of the one loop eigenvalues and classical energy minimality (see Appendix B.3), for the case $\mathrm{A}_{4}^{a} \neq 0$.

Having made the comparison for the case $\overline{\mathrm{A}}_{4}^{a}=0$, we find it clear that the existence of negative eigenvalues as in Eq. (73) leads immediately to the conclusion that the theory is also classically unstable as illustrated by sikivie, Weiss and Chang for the constant magnetic field. 33-35

Let us return to the problem of dealing with the negative eigenvalues. Eq. (74) has been written as a product of integrals. Each integral is equal to the area under one of the curves in Fig. 2. The quadratic approximation can be pictured as the product of the areas under the curves in Fig. 3. For values of $\mathrm{k}^{2}>\mathrm{m}^{2}$, the curves are raussian and the area is finite. For $k^{2}<m^{2}$ (the top set of curves in Fig. 3), the area under each curve diverges. Now, if we interpret the existence of the negative eigenvalues as indicating an instability of the theory when expanded around a "bad" field configuration, can the quadratic approximation tell us anything more about the nature of this instability? Imagine that we have two different theories, characterized by the solid curves in Fig. 4. If in each case we expand about a configuration corresponding to the local minimum of the integrand of 
Eq. (74) with $k^{2}=0$ (corresponding to minima in Fig. 4), then our theory is more unstable (we have farther to fall) for curve II than for curve $I$, where $\mathrm{m}_{I I}^{2}>\mathrm{m}_{I}^{2}$. We would like to somehow "regularize" the functional integral over the negative eigenvalue modes in order to gain some information about the degree of instability.

If we formulate the functional integral in Minkowski space, rather than in Euclidean space, then it is necessary to regularize the theory by taking the prescription of adding an infinitesimal term, $i \varepsilon \phi^{2}$, to the Lagrangian density in such a way as to insure causal boundary conditions. 40 It turns out that this procedure also regularizes the infinities due to the presence of tachyons in the theory, and produces an imaginary contribution to $\Gamma$. If we continue a non-tachyonic theory to Euclidean space, the is term is usually dropped, for the theory is now well defined mathematically. However, in the presence of tachyonic modes, the loop approximation breaks down. We would like to define some alternative "regularization" for the tachyonic modes which would reproduce the imaginary part of $\Gamma$. The advantage of doing so in Euclidean space will be to help us better understand the physical meaning of the imaginary contribution.

In the work due to Callan and Coleman on the decay of the false vacuum $1,36,37$ and in that due to Langer ${ }^{38}$ on nucleation, a similar problem arises. There, the expansion 
around the metastable minimum gives a real contribution. They are also interested in calculating a non-perturbative quantum mechanical correction due to the possibility of quantum tunneling to the lower vacuum configuration. To this effect they expand the integral around the Euclidean "bounce" finite action solution to the classical equations. They also encounter negative eigenvalues due to an expansion around a maximum of the action, although not a maximum of the potential. In order to "regularize" these theories, they refer to another well defined theory such that the analytic continuation of some parameter gives the physical theory. Thus to calculate a contribution due to the negative eigenvalue, they write down a steepest descent integral in the well defined theory and, as they analytically continue to the physcial theory, they also analytically continue both the path of integration and the boundary conditions into the complex field plane in such a way as to keep the theory well defined. This just corresponds to picking the "paths of steepest descent". The ad hoc feature of this procedure is that, to keep the theory finite, the boundary conditions are also continued, so that in effect we are computing a different functional integral and hence have "regularized" the theory. The justification in this case comes from the physical system. We are expanding around a metastable state and the imaginary 
part computed should represent the decay rate for this state. In fact, for a few examples taken from quantum mechanics, the decay rates calculated in this way agree with those calculated from more conventional methods. There is, however, no justification that this procedure and interpretation are correct in general. With the hope that this mathematical procedure does have some general physical significance, we will apply it to our problem.

First we shall discuss our sample theory, Eq. (74) with $\mathrm{k}^{2}=0$. If we write down the Lagrangian for a $\phi^{4}$ theory with $\mathrm{m}^{2}>0$, then the tachyonic theory can be obtained by continuing $+m^{2}$ to $-m^{2}$. The theory with $+m^{2}$ has a saddle point at the origin in $\phi$ space and has two other saddle points along the imaginary axis in $\phi$ space (see Fig. 5a). The saddle point at the origin is a maximum of the integrand along the real axis and hence the quadratic integral is well defined. Those saddlepoints on the imaginary axis are higher maxima. Thus, if we rotate the complex plane, as in Fig. 5b, we will reproduce the double humps in Figs. 2 and 4 . Our procedure then is to rotate the contour as we rotate in the complex plane so as to always remain on the path of steepest descent from the saddlepoint. Now depending on how we rotate our contour we will either go up or down the imaginary axis. There are a number of ways in which to decide. If we keep the choice arbitrary, then at the end of the calculation, conservation 
of probability (unitarity) will demand a definite sign for the calculated imaginary part. Alternatively, we may keep the $i \varepsilon \phi^{2}$ contribution to the Lagrangian in Minkowski space and, upon doing our calculations in Euclidean space, the pole structure of the negative eigenvalue contributions will be determined by the sign of $\varepsilon$. In regard to this question we have also found that instead of making the continuation noted above, we can rotate $\phi \rightarrow e^{i \pi / 4} \phi$ so that, keeping the iع $\phi^{2}$ contribution, the integrand changes:

$$
\exp \left(|\lambda| \dot{\phi}^{2}+i \varepsilon \phi^{2}\right) \rightarrow \exp \left(i|\lambda| \phi^{2}-\varepsilon \phi^{2}\right)
$$

This integrand is identical to the type we would have if we had formulated the theory in Minkowski space. If $\phi \rightarrow e^{-i \pi / 4} \phi$, then the $\varepsilon$ contribution would diverge. For the present we shall proceed as first mentioned and will decide the direction of rotation by demanding unitarity. Therefore, we shall write these negative eigenvalue contributions as

$$
\pm \int_{-i \infty}^{+i \infty} d \phi_{k} \exp \left[-\frac{1}{2} \int_{-m}^{m} d k \phi_{k}\left(k^{2}-m^{2}\right) \phi_{k}\right] \simeq \pi_{k=-m}^{m} \pm i \int_{-\infty}^{+\infty} d \phi_{k} \exp \left[-\frac{1}{2} \Delta_{k}\left(m^{2}-k^{2}\right) \phi_{k}^{2}\right]
$$

which gives a finite imaginary result.

A few comments are in order. In the theory of nucleation there is an extra factor of $1 / 2$. In that problem we integrate over all of function space up to the negative 
eigenvalue mode and then deform the contour to go over only half of the Gaussian. In our case there is no metastable region. We expand around a local maximum of the potential. Hence, for the integral to remain well defined, we must integrate over the whole Gaussian. second, the contribution calculated by Callan and coleman is of exponentially small order and, as already noted, has a non-perturbative origin, while our calculation is done in perturbation theory. Third, in the metastable case some physical argument in terms of quantum tunneling can be given for performing such a continuation. In our case, expanding about a local maximum, no such argument exists. Our problem is more in analogy with Schwinger's calculation of an amplitude for pair production in the presence of a constant electric field, although his definition of the effective action does not involve path integrals. ${ }^{6}$ we will therefore perform the analytic continuation in analogy with the theory of metastability, study our result and finally compare it with that of schwinger.

First let us examine the expression we get upon performingour analytic continuation to see if it contains the information we desire. For the case of our sample theory, the two inverted Gaussians (dashed lines) in Fig. 4 are replaced in the analytic continuation by the 
solid curves in Fig. 6, where the integrand (now imaginary) is plotted versus the continued $\phi$ axis. Since $\phi=0$ is a saddle point in the complex plane, and a minimum along the real axis, it is a maximum along the imaginary axis. If $\mathrm{m}_{\text {II }}^{2}>\mathrm{m}_{I}^{2}$, then the $\mathrm{k}=0$ curve for II blows up faster along the real axis, but falls off faster along the imaginary axis than the $k=0$ curve for I. Eq. (82) is equal to the product of the (imaginary) areas under the solid curves, above and including the solid line in Fig. 6 . It appears then that the contribution from the theory with the larger tachyon mass, 'mI' will give a larger contribution than the theory with the smaller mass, $\mathrm{m}_{I}$. However, what is of primary interest is a calculation of $\Gamma_{E}$ which is proportional to the logarithm of the functional integral. As mentioned in section 3 , the logarithm of $\mathrm{Eq} .(82)$ is

$$
\int_{-m}^{m} d k \ln \pm i\left(m^{2}-k^{2}\right)^{-\frac{1}{2}}=-\frac{1}{2} \int_{-m}^{m} d k \ln \left(k^{2}-m^{2}\right)
$$

which is just the same form as we get for modes in which $k^{2}>m^{2}$. Therefore our analytic continuation for the negative eigenvalue modes will reproduce the second term in Eq. (71) for the case $n=0$, for all values of $\mathrm{k}^{2}=\mathrm{k}_{4}^{2}+\mathrm{k}_{\mathrm{z}}^{2}$. The logarithm in Eq. (83) can be rewritten as 


$$
\ln \pm i\left(m^{2}-k^{2}\right)^{-\frac{1}{2}}= \pm i \frac{\pi}{2}+\ln \left(m^{2}-k^{2}\right)^{-\frac{1}{2}}
$$

Thus our analytic continuation has produced a real and an imaginary contribution to $\Gamma_{E}$, with the imaginary contribution proportional to imm. Thus in Fig. 6 , for $\mathrm{m}_{I I}$ the contribution to the imaginary part is larger due to the larger range of integration, or the existence of a larger number of tachyonic curves above curve II. Thus the imaginary contribution to the effective action is clearly larger for larger tachyonic masses, or greater instabilities. Let us now calculate the real and imaginary contributions of the "regularized" tachyonic modes in the constant color magnetic field example. The term we wish to calculate is

$$
N \rightarrow \frac{g B}{2 \pi} \int d^{4} x \int_{-\infty}^{+\infty} \frac{d k_{4}}{2 \pi} \int_{-\infty}^{+\infty} \frac{d k_{z}}{2 \pi} 2 \ln \left(k_{4}^{2}+k_{z}^{2}-g B\right)^{-\frac{2}{2}}
$$

where we have rotated $\tau$ back to Minkowski space. Now for $k_{z}^{2}>g B$, the $k_{4}$ integral is of the form $\int_{-\infty}^{+\infty} d k_{4} \ln \left(k_{4}^{2}+x^{2}\right)^{-\frac{1}{2}}$ which has already been done in section 3. This leads to a real contribution:

$$
-\frac{1}{2} \frac{g B}{2 \pi} \int d^{4} x \int_{\sqrt{g B}}^{\infty} \frac{d k z}{2 \pi} 4\left[\sqrt{k_{z}^{2}-g B}-\sqrt{k_{z}^{2}}\right]
$$

For $k_{z}^{2}<g B$, the $k_{4}$ integral is of the form

$$
\int_{-\infty}^{+\infty} d k_{4} \ln \left(k_{4}^{2}-x^{2}\right)^{-\frac{1}{2}}
$$


where $x^{2}=g B-k_{z}^{2}>0$. For $k_{4}^{2}>x^{2}$, there are still positive eigenvalue contributions. For $k_{4}^{2}<\mathrm{x}^{2}$,

$$
\int_{-x}^{x} d k_{4} \ln \pm i\left(x^{2}-k_{4}^{2}\right)^{-\frac{1}{2}}=\int_{-x}^{x} d k_{4}\left[ \pm i \frac{\pi}{2}+\ln \left(x^{2}-k_{4}^{2}\right)^{-\frac{1}{2}}\right]
$$

where the \pm sign is the same as in Eq. (83) and depends upon which way we have rotated the contour for the functional integral. Therefore, for $k_{z}^{2}<g B$ we have

$$
\pm \pi i \sqrt{g B-k_{z}^{2}}+\int_{-\infty}^{\infty} d k_{4}\left[\ln \left|k_{4}^{2}+k_{z}^{2}-g B\right|^{-\frac{1}{2}}-\ln \left(k_{4}^{2}+k_{z}^{2}\right)^{-\frac{1}{2}}\right]
$$

The last term in brackets comes from the normalization $\mathrm{N}$. The integral above vanishes identically. That is, the real part of the negative eigenvalue contribution exactly cancels the real contribution from the positive eigenvalue modes for which $\mathrm{k}_{\mathrm{z}}^{2}<\mathrm{gB}$ ! In analogy to the discussion by Langer, ${ }^{38}$ the real part of the effective action in the tachyonic sector of the theory as calculated above is the effective action due to the "metastable" phase. In Section 3 we interpreted the real part as a change in the zero point energy. For $\mathrm{k}_{\mathrm{z}}^{2}<\mathrm{gB}$, the energy contribution $k_{0}=\sqrt{k_{z}^{2}-g B}$ is imaginary and hence only contributes to 
the imaginary part of $\Gamma$. For $k_{z}^{2}>g B$, however, there is a nonvanishing contribution to the real part.

If we complete the evaluation of the imaginary part, we find

$$
i \Gamma_{\text {imag. }}= \pm i \zeta \frac{g^{2} B^{2}}{8 \pi} \int d^{4} x
$$

Note that the contribution to the imaginary part of the effective lagrangian density is finite as it stands, i.e., before we renormalize the ultraviolet divergence of the theory. This is in keeping with the relationship of the instability to the classical analysis. To choose the sign of the imaginary part, we consider the vacuum generating functional $W(J)$. In the absence of a source, as shown in Eqs. (2l) and (24), we can simply relate $\Gamma$ and $W$ :

$$
\Gamma(\bar{A})=W(0)
$$

so that

$$
\left|<0^{+}\right| 0^{-}>\left.\right|^{2}=\left|\exp \frac{i}{h} \Gamma\right|=\exp \left(-2 \Gamma_{\text {imag. }} / \hbar\right) .
$$

According to Schwinger's interpretation, ${ }^{6}$ this is the probability that no pair creation occurs during the history of the field. 
From Eq. (88), we see that, to conserve probability,

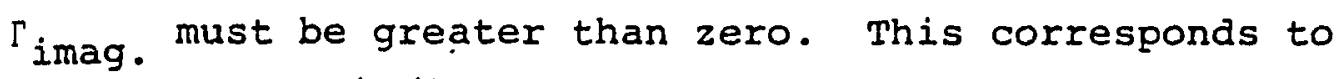
letting $\phi \rightarrow e^{+i \pi / 2} \phi$ in our analytic continuation or we have rotated our contour counter-clockwise.

Note also that to make this connection to Schwinger's work, it is imperative to be able to relate the effective action $\Gamma$ to the vacuum generating functional $W$, as was done in section 2 .

As to the imaginary part itself, Schwinger defines $2 \operatorname{Im} L$ as the probability, per unit time, per unit volume of a pair being created by the external field. There are thus two interpretations of our calculation. The first is as above, that in the presence of an external color magnetic field, the vacuum is unstable and will pair produce. If we consider Eq. (88), then $\mathrm{g}^{2} \mathrm{~B}^{2} / 4 \pi$ is the probability per unit four volume of pair production. If this probability is of order one, the magnetic field cannot be maintained without radiating. For a four volume larger than $\left(g^{2} B^{2}\right)^{-2}$, we may interpret our result as meaning that it would be impossible to maintain a constant magnetic field at all. In a smaller four volume, the contribution of the negative eigenvalues decreases, in agreement with the conclusion of Chang and weiss ${ }^{34}$ that the classical unstable mode will not exist in a length $I$ smaller than $(g B)^{-\frac{1}{2}}$. In a classical stability analysis, they find that the classical unstable modes fall off exponentially in space. They define a three 
volume per unit unstable mode proportional to $(g B)^{-3 / 2}$. Chang and Weiss make the analogy between $\sqrt{g B} L$ and the Reynolds number in fluid mechanics. This is also reminiscent of the quantum fluid model of Nielsen, Olesen and Ambjorn. 27-31 Perhaps the QCD vacuum is composed of "pockets" of constant magnetic field of size $L<(g B)^{-1 / 2}$.

The second interpretation of the calculation is that we are trying to approximate the effective action for the case of no external fields but that, under the assumption of a spontaneously generated constant magnetic field, our theory is unstable. In this regard note that for $E_{i}^{a}=0$, the effective Hamiltonian density equals minus the effective Lagrangian density. The effective Hamiltonian thus has an imaginary part, indicating that the state we are studying is not to be found in our Hilbert space and is unstable. 36 From our discussion about the source of the instability as due to the expansion about a local maximum, it is interpreted that for small values of the imaginary part, the real part of $\Gamma$ per unit time may still be a fair approximation to the energy functional. This is also in agreement with the discussion of Ref. 27-31, for the presumed minimum of the renormalization group improved effective action occurs for small values of the magnetic field. 


\section{DISCUSSION AND SUMMARY}

We have derived the gauge invariant effective action functional of 't Hooft ${ }^{15}$ from a formulation of the vacuum to vacuum transition amplitude in the background field gauge. Thus the definition of the gauge invariant effective action for non-Abelian gauge theories is made consistent with the definition for non-gauge theories. Also, this connection allows us to utilize schwinger's interpretation of the imaginary part of the Lagrangian density in the absence of sources. 6 Further, we have explicitly demonstrated the gauge invariance properties of the effective action and, noting this, have been able to justify the hypothesis of Nielsen and Olesen ${ }^{16}$ that the unrenormalized one-loop approximation for the effective action, in the presence of an external field, can be written as minus the change in the zero point energy of the theory due to the presence of an external field. We have justified this result for the case when the external field is static and $\overline{\mathrm{A}}_{0}^{a}=0$, although we suggest that it may be extended to $\overline{\mathrm{A}}_{0}^{a} \neq 0$. In particular we justify the use of this hypothesis for the case of a constant color magnetic field, at least for modes for which the functional integral is well defined (no negative eigenvalues in the Euclidean space formulation). We have also noted that the physical field in 't Hooft's definition is not the customary vacuum expectation of the quantum field, but differs by the expectation value of the quantum field relative to a new "shifted" vacuum. 
We have studied the "semi-classical" stability of sample theories by formulating the functional integral in Euclidean space and noting the existence of negative eigenvalues when negative mass squared terms are present in the Euclidean action. By examining the Euclidean formulation of the theory, we have been able to compare the "quantum instability" to instabilities arising in a classical analysis. In this regard, we have been able to explicitly demonstrate the equivalence of certain classical and quantum mechanical stabilities and instabilities and, for example, have been able to equate the classical instability of the constant color magnetic field demonstrated by sikivie 33 and chang and Weiss, ${ }^{34}$ to the quantum instability noted by Nielsen and olesen, ${ }^{16}$ Yildiz and $\operatorname{cox}^{22}$ and Falomir and schaposnik. 18

As far as the evaluation of the effective action when negative eigenvalues are present, we have referred to the prescription of Callan and Coleman in the "Decay of the False Vacuum" 36 and have "regularized" the negative eigenvalue modes by analytically continuing the functional integral into the complex field plane, where the direction of rotation is determined by demanding causal boundary conditions, as in the Minkowski space formulation, or by demanding unitarity. This apparently ad hoc procedure reproduces the results of calculating the real and imaginary parts of the effective action in Minkowski space. This procedure resembles that of "Wick rotating" ordinary integrals 
and although the analogy is not complete, the equivalence of the Minkowski space calculation encourages us to speculate that there is perhaps some deeper mathematical meaning to the analytic continuation and perhaps some kind of formal functional wick rotation may indeed be formulated!

We have calculated the imaginary part of the effective action for the case of the constant magnetic field and have connected its interpretation to that of pair production in a constant electric field, thereby showing agreement with Chang and Weiss ${ }^{34}$ on the existence of a minimal "length" for the unstable mode. We have also shown agreement for the constant magnetic field case with the interpretation of Nielsen and olesen 16 that the imaginary part represents an imaginary energy density and hence the existence of lower energy stable configurations.

By calculating the one-loop term as the change in the zero point energy of the theory, we can easily separate the real and imaginary parts of the effective action. Our procedure of analytically rotating the field contour in Euclidean space reproduces the naive calculation obtained by ignoring the existence of negative eigenvalues and demonstrates that the imaginary part is a sum over zero point energies which are totally imaginary. It should be noted that the imaginary part of the effective Lagrangian density is finite and does not suffer from the ultraviolet divergences which occur for all of the other momentum integrations. 
In most of this work we have neglected the renormalization of physical parameters needed to regulate the above mentioned divergences. Many renoramlization schemes can be found in the literature and the renormalization for the case of the constant magnetic field is shown in Refs. 16, 18 and 22. Premature renormalization, though, can obscure the essential simplicity of the one-loop approximation as a change in the zero point energy. 
APPENDICES 


\section{APPENDIX A: COMPARISON OF CLASSICAL MINIMALITY \\ AND POSITIVE DEFINITENESS OF THE ONE- \\ LOOP EIGENVALUES FOR YANG MILLS FIELDS}

The classical Hamiltonian is

$$
H=\frac{1}{2} \int \dot{d}^{3} \times\left[\left(E_{i}^{a}\right)^{2}+\left(B_{i}^{a}\right)^{2}\right] .
$$

We wish to study the sufficient stability criterion of minimizing the energy $H$, subject to the constraint of Gauss law:

$$
\vec{\nabla} \cdot \vec{E}^{a}-g c^{a b c} \vec{A}^{b} \cdot \vec{E}^{c}=0 \text {. }
$$

Following a derivation by Jackiw and Rossi, 47 we therefore extremize Eq. (89) subject to Eq. (90) by introducing the Lagrange multiplier $A_{0}^{a}$. Making the ansatz that the minimal configuration is static, we have

$$
\begin{aligned}
\frac{\partial E^{i a}}{\partial t} & =0=\frac{\delta E}{\delta A_{i}^{a}}=\varepsilon^{i j k}{ }_{j} B_{k}^{a}-g c^{a b c} \varepsilon^{i j k_{A} b_{j} c} \\
-\frac{\partial A_{k}}{\partial t} & =0=\frac{\delta E}{\delta E_{i}^{a}}=E^{i a}+\nabla^{i} A_{0}^{a}-g c^{a b c_{A} i b_{A} c}
\end{aligned}
$$

where $E$ is the constrained energy density. The second variation of the constrained energy gives 


$$
\begin{gathered}
\frac{\delta^{2} E}{\delta E_{i}^{c} \delta A_{j}^{a}}=-\frac{\delta^{2} E}{\delta A_{i}^{c} \delta E_{j}^{a}}=-\delta^{i j g c^{a b c} A_{0}^{b}} \\
\frac{\delta^{2} E}{\delta E_{i}^{a} \delta E_{j}^{b}}=\delta^{i j} \delta^{a b} \\
\frac{\delta^{2} E}{\delta A_{i}^{C} \delta A_{j}^{a}}=\frac{1}{2} \frac{\delta^{2}\left(B_{k}^{b}\right)^{2}}{\delta A_{i}^{c} \delta A_{j}^{a}}=\varepsilon^{i k m_{\varepsilon} n k j_{D} c b_{D}^{b a}}-\varepsilon_{n}^{i k j} g c c b a_{B}^{b} .
\end{gathered}
$$

The first term above is of gyroscopic nature and leads to the complications noted in Ref. 47.

Now we also expand the action to second order around a static solution of the classical equations of motion in Euclidean space. The action is

$$
S=\frac{1}{2} \int d^{4} \times\left[\left(E_{i}^{a}\right)^{2}-\left(B_{i}^{a}\right)^{2}\right]
$$

with $E_{i}^{a}$ and $B_{i}^{a}$ as defined in $E^{a}$. (45). In Euclidean space

$$
S_{E}=\frac{1}{2} \int d^{4} x_{E}\left[\left(E_{i}^{a}\right)^{2}+\left(B_{i}^{a}\right)^{2}\right]
$$

where now $E_{i}^{a}=F_{i 4}^{a}$. If we expand around static solutions of $\left(\delta S_{E} / \delta A_{\mu}^{a}\right)_{\bar{A}}=0$, then the quadratic approximation to the functional integral has as its integrand the exponential of

$$
-\frac{1}{2} A^{\mu c}\left[\frac{\delta^{2} S_{E}}{\delta A^{\mu c} \delta A^{v a}}-\frac{1}{\alpha}\left(D_{\mu} D_{V}\right)^{c a}\right]_{\bar{A}} A^{\nu a}+\text { Ghost Terms. }
$$

The ghost term always has positive definite eigenvalues. 
The second term is the gauge fixing term in the covariant background field gauge, and all terms in the brackets are evaluated at the static classical field $\bar{A}_{\mu}^{a}$. Therefore, we desire that the bracketed operator is positive definite. Call this operator $G_{\mu \nu}^{c a}$. We have already shown (see Eq. (50)) that in the gauge $\alpha=1$ we have

$$
G_{\mu \nu}^{c a}=\delta_{\mu \nu}\left(D_{\alpha} D^{\alpha}\right)^{c a}-2 g c^{c b a} F_{\mu \nu}^{b} .
$$

Thus

$$
\dot{G}_{00}^{c a}=\left(D_{\alpha} D^{\alpha}\right)^{c a}=-\left(D^{\alpha} D^{\alpha}\right)^{c a}
$$

which is positive semi-definite while

$$
G_{4 i}^{c a}=-G_{i 4}^{c a}=-2 g c^{c b a} F_{4 i}^{b}=2 g c^{c b a} E^{i b}
$$

and

$$
\begin{aligned}
G_{i j}^{c a} & =\delta_{i j}\left(-D^{\alpha} D^{\alpha}\right)^{a c}-2 g c^{c b a} F_{i j}^{b} \\
& =\frac{1}{2} \frac{\delta^{2}\left(E_{k}^{b}\right)^{2}}{\delta A^{i c} \delta A^{j a}}+\frac{1}{2} \frac{\delta^{2}\left(B_{k}^{b}\right)^{2}}{\delta A^{i C} \delta A^{j a}}-\left(D_{i} D_{j}\right)^{c a} . \\
& =-\delta_{i j}\left(D^{4} D^{4}\right)^{c a}+\frac{1}{2} \frac{\delta^{2}\left(B_{k}^{b}\right)^{2}}{\delta A^{i C} \delta A^{j a}}-\left(D_{i} D_{j}\right)^{c a} .
\end{aligned}
$$


In general, the comparison with classical minimality is complicated. Classically we have a gyroscopic term, and quantum mechanically there are cross terms between $A_{4}^{a}$ and $A_{i}^{a}$. If we restrict ourselves to the gauge choice $A_{0}^{a}=0$, however, things, simplify. The classical analysis reduces to

$$
\frac{i}{2} \frac{\delta^{2}\left(B_{k}^{b}\right)^{2}}{\delta A_{i}^{c} \delta A_{j}^{a}}>0
$$

and positive definiteness of the one-loop eigenvalues requires only that

$$
-\delta_{i j}\left(D^{4} D^{4}\right) c a+\frac{1}{2} \frac{\delta^{2}\left(B_{k}^{b}\right)^{2}}{\delta A^{i C} \delta A^{j a}}-\left(D_{i} D_{j}\right)^{c a}>0 .
$$

The first term in the latter expression is positive semi-definite. The second term is the same as in the classical expression, while the third is the gauge fixing term. To compare with the classical case, let us use the remaining time independent gauge freedom to fix the classical fluctuations with $D_{i}^{a b}(\bar{A}) \delta A^{i b}=0$. Now in the expansion of the constrained Hamiltonian there are terms like $I / 2\left(\delta A^{i c}\right)\left(\delta^{2} E / \delta A^{i c} \delta A^{j a}\right) \delta_{\bar{A}} \delta a$. If we use the above gauge choice, we may add a term $\delta A^{i C}\left[D_{i}(\bar{A}) D_{j}(\bar{A})\right]$ ca $\times \delta A^{j a}$ to the above expression so that $\left(\delta^{2} E / \delta A^{i c} \delta A^{j a}\right)+$ $(1 / 2)\left(\delta^{2}\left(B_{k}^{b}\right)^{2} / \delta A^{i c} \delta A^{j a}\right)-\left(D_{i} D_{j}\right)^{c a}>0$. Comparing this 
to the one-loop expression, we conclude that classical stability leads to the positivity of the one loop eigenvalues and that negativity of one loop eigenvalues leads to classical instability (modulo exceptions mentioned in Section 4).

The case of gyroscopic contributions is analyzed in Appendix B.3. For this case the above statements holds provided we substitute "energy minimality" for "classical stability". We have not reproduced this analysis for YangMills fields with $\overline{\mathrm{A}}_{0}^{\mathrm{a}} \neq 0$. However, the results of this section and of Appendix B.3 suggest that our statement may hold for that case, too. 


\section{APPENDIX B: GYROSCOPIC STABILITY}

\section{B.1. Classical Stability}

We shall take the following definition of classical stability. 34,47 Let us expand the classical Euler-Lagrange or Hamilton equations in small oscillations around particular solutions. This defines a new set of equations of motion for the classical fluctuations. If the time dependence of the fluctuations is such that there is an exponential growth in time, then we have expanded about an unstable configuration. If, on the other hand, the fluctuations are oscillatory in time, we have expanded around a locally stable configuration. In Reference (47), it is also stated that a polynomial growth in time is not a sign of instability, but is a sign of a degeneracy in the small oscillation eigenfrequencies.

In the following and in the text our analysis is limited to expansions around static or time-independent solutions of the classical equations. Also, in order to differentiate between the criteria of energy minimality and gyroscopic stability (to be defined below), it is convenient to work in the Hamiltonian formulation of the theory. (To formulate the theory covariantly, however, it is far easier to use the Lagrangian formulation.) In the following we shall follow the notation and presentation of Reference (47).

Let us imagine a system described by a Hamiltonian $H(P, Q)$ which is a function of $\mathrm{N}$ canonical momenta, $\mathrm{P}$, and $\mathrm{N}$ canonical 
64

coordinates, $Q$. If the system is time translation invariant then the Hamiltonian is time-independent and represents the conserved energy of the theory.

Hamilton's equations for the theory are

$$
\dot{\mathrm{P}}_{i}=-\frac{\partial H(\mathrm{P}, \mathrm{Q})}{\partial \mathrm{Q}_{i}}
$$

$$
\dot{Q}_{i}=\frac{\partial H(P, Q)}{\partial P_{i}}
$$

To expand the theory around static solutions, let

$$
P_{i}=P_{i s}+\delta P_{i} \quad Q_{i}=Q_{i s}+\delta Q_{i}
$$

where

$$
\dot{P}_{\text {is }}=\dot{Q}_{\text {is }}=0 \text {. }
$$

From Equations (99) it is clear that these solutions represent extrema of the Hamiltonian. Expanding Equations (99) we get

$$
\begin{aligned}
& \delta \dot{P}_{n}=-\frac{\partial^{2} H}{\partial Q_{m_{s}} \partial Q_{n_{s}}} \delta Q_{m}-\frac{\partial^{2} H}{\partial P_{m_{s}} \partial Q_{n_{s}}} \delta P_{m} \\
& \delta \dot{Q}_{n}=\frac{\partial^{2} H}{\partial P_{m_{s}} \partial P_{n}} \delta P_{m}+\frac{\partial^{2} H}{\partial Q_{m_{s}} \partial P_{n}} \delta Q_{m}
\end{aligned}
$$

As an alternative method of calculation, expand $H(P, Q)$ about static solutions. Then

$$
\begin{aligned}
H(P, Q) & =H\left(P_{S} \cdot Q_{S}\right)+\frac{1}{2} \delta P_{n} T_{n m} \delta P_{m}+\delta P_{n} G_{n m} \delta Q_{m} \\
& +\frac{1}{2} \delta Q_{n} V_{n m} \delta Q_{m}+\ldots
\end{aligned}
$$


The linear terms in Equation (102) vanish since the static field is an extrema of the Hamiltonian. This can be rewritten as

$$
H(P, Q)=H\left(P_{S}, Q_{S}\right)+\frac{1}{2} \tilde{x} H \times \ldots
$$

where

$$
H=\left(\begin{array}{cc}
\mathrm{T} & G \\
\tilde{G} & V
\end{array}\right), X=\left(\begin{array}{l}
\delta P \\
\delta Q
\end{array}\right)
$$

$H$ is a $2 \mathrm{~N} x 2 \mathrm{~N}$ matrix. $X$ is a $2 \mathrm{~N}$ column matrix, and $\tilde{X}$ is its transpose. $H$ is then the second variation of $H$ evaluated at $\left(P_{S}, Q_{S}\right)$, and Equation $(101)$ can be represented as

$$
H x=i n \dot{x}
$$

where

$$
n=\left(\begin{array}{cc}
0 & -i I \\
i I & 0
\end{array}\right)
$$

and $I$ is the $N \times N$ unit matrix. To analyze the stability of the static solutions, we let $x=e^{-i \omega t} x$. Then from Equation

$$
H x=\omega n x
$$

$x$ is called the simplectic eigenvector of $H$ with simplectic eigenvalue $\omega$. Liapunov's Theorem ${ }^{47}$ states that for stability, the w's must be real. If we diagonalize the matrix $H$ we have for stability

$$
\operatorname{det}(H-\omega n)=0, \omega \text { real. }
$$


Now

$$
x^{+} H x=\omega x^{+} n x
$$

Since $H$ is real symmetric (hermitian), the left hand side is real. Also $n$ is hermitian, so $x^{+} n x$ is real. Therefore, for instability both $x^{+} H x$ and $x^{+} \eta x$ must be 0 .

In the above we have expanded the Hamiltonian (energy) about its extrema. If we demand that the second variation of the energy is positive definite, that is

$$
\operatorname{det}(H-\lambda I)=0, \lambda>0,
$$

then we have expanded about a minimum of the energy. It is clear that Equation (110) satisfies our criteria for stability, Equation (108), because if $H$ is positive definite, then from Equation (109), $\omega$ is real. Therefore, energy minimality implies classical stability. However, it is not a necessary condition. $\omega$ can be real without $H$ being positive definite. This alternative condition is labeled gyroscopic stability. In order to simplify the comparison between Equation (108) and Equation (110), we shall somewhat specialize our example. Assume that the kinetic energy is positive definite and normalized to the identity. The off diagonal gyroscopic or coriolis term, $G$, is taken to be anti-symmetric since if we derive the theory from the Lagrangian formalism, any symmetric part in $G$ corresponds to a total time derivative and may be dropped. (Note: if we are dealing with an effective theory which treats couplings to an external dissipative system by using friction type terms, then these terms are not derived 
from a Lagrangian and may not be dropped. Also the existence of non-trivial temporal boundary conditions could also contribute to a symmetric part of G.) Modulo the last remarks, we have

$$
\begin{aligned}
& H=\left(\begin{array}{cc}
I & G \\
-G & V
\end{array}\right) \\
& \tilde{G}=-G, \tilde{V}=V .
\end{aligned}
$$

Equation (107) becomes

$$
[(i \omega+G)(i \omega+G)+v] \delta Q=0
$$

and Equation (108) is

$$
\operatorname{det}\left(2 i \omega G+G^{2}+V-\omega^{2} I\right)=0, \omega \text { real. }
$$

For minimality we require positivity of $H$ which is equivalent to positivity of $\tilde{M} H M$ where

$$
M=\left(\begin{array}{cc}
I & -G \\
0 & I
\end{array}\right), \tilde{M} H M=\left(\begin{array}{cc}
I & 0 \\
0 & G^{2}+V
\end{array}\right)
$$

Equation (110) is then satisfied by

$$
\operatorname{det}\left(G^{2}+V-\lambda I\right)=0, \lambda>0 .
$$

Therefore, comparing Equation (113) with Equation (115), if $G=0$, then $\omega^{2}$ equals $\lambda$ and $\omega$ real implies $\lambda$ positive. If $G \neq 0$, however, the two conditions are different and we have the possibility of stable static solutions which do not minimize the energy. (Note again that friction or dissipative forces could render these solutions unstable.) As shown in Ref. (47), the signature for gyroscopic stability is the 
occurrence, arbitrarily close to the static solution, of harmonic fluctuations which lower the energy. If instead, the solutions grow exponentially in time, then we have instability. Examples of gyroscopically stable configurations can be found in tops, gyroscopes, and planetary configurations. In all of these examples, it is the occurrence of a non-zero value of a conserved quantity (angular momentum) which allows for the non-zero value of the gyroscopic term and hence for gyroscopic stability.

\section{B.2. The Vertical Top}

As an example of gyroscopic stability consider the Hamiltonian, 47

$$
\mathrm{H}=\frac{1}{2}\left(\mathrm{P}_{1}^{2}+\mathrm{P}_{2}^{2}\right)+\frac{L}{2}\left(\mathrm{P}_{1} \mathrm{Q}_{2}-\mathrm{P}_{2} \mathrm{Q}_{1}\right)+\frac{1}{2} \Omega^{2}\left(\mathrm{Q}_{1}{ }^{2}+\mathrm{Q}_{2}{ }^{2}\right) .
$$

This is an effective Hamiltonian for a symmetrical top. The canonical coordinates are the Euler angles $(\theta, \phi, \psi), 54$ and we are restricting $\theta$, the angle between the vertical and the symmetry axis, to be very smali $(\sin \theta \sim \theta)$. This nearly vertical top has two cyclic variables; $\mathrm{P}_{\phi^{\prime}} \mathrm{P}_{\psi}$. In Equation we have explicitly fixed $\mathrm{P}_{\psi}$, the angular momentum about the symmetry axis (body fixed axis) to equal L. Also 55

$$
Q_{1}=\theta \cos \phi, Q_{2}=\theta \sin \phi
$$

and

$$
\Omega^{2}=L^{2} / 4-M g \ell
$$


where $\mathrm{Mgl}$ is the potential energy of the center of mass. $I_{1}$ ' the symmetric moment of inertia, has been set equal to 1 . If we expand Equation (116) around $P_{i}=Q_{i}=0$ (a vertical top), then Equation (116) itself represents the quadratic approximation. Therefore

$$
H=\left(\begin{array}{cccc}
1 & 0 & 0 & L / 2 \\
0 & 1 & -L / 2 & 0 \\
0 & -L / 2 & \Omega^{2} & 0 \\
L / 2 & 0 & 0 & \Omega^{2}
\end{array}\right)
$$

so that

$$
\begin{aligned}
& G=\left(\begin{array}{cc}
0 & L / 2 \\
-L / 2 & 0
\end{array}\right) \\
& V=\left(\begin{array}{cc}
\Omega^{2} & 0 \\
0 & \Omega^{2}
\end{array}\right) .
\end{aligned}
$$

In this case Equation (115), the minimality condition, gives

$$
\left|\begin{array}{cc}
\Omega^{2}-L^{2} / 4-\lambda & 0 \\
0 & \Omega^{2}-L^{2} / 4-\lambda
\end{array}\right|=0
$$

or

$$
\lambda=\Omega^{2}-L^{2} / 4
$$

Equation (114), the gyroscopic condition, gives

$$
\left|\begin{array}{cc}
\Omega^{2}-L^{2} / 4-\omega^{2} & i \omega L \\
-i \omega L & \Omega^{2}-2 / 4-\omega^{2}
\end{array}\right|=0
$$

or

$$
\omega=\Omega \pm L / 2 \text {. }
$$


For energy minimality, $\lambda>0$, and we then require that

$$
\Omega>L / 2 \text {. }
$$

This cannot be satisfied since $\Omega^{2}=L^{2} / 4-$ Mgl. For gyroscopic stability we require that $\omega$ is real or that

$$
\Omega^{2}>0
$$

The top is then gyroscopically stable if $L^{2} / 4>\mathrm{Mgl}$. Therefore, if $L$ is sufficiently large, the presence of the gyroscopic term in the Hamiltonian stabilizes an otherwise unstable configuration.

\section{B.3. The One Loop Effective Action}

Let us now examine the effect of gyroscopic terms upon the interpretation in terms of classical stability of the positivity of the eigenvalues arising in the one loop Euclidean effective action calculation. Referring again to the Hamiltonian in Equation (116), Hamilton's equations give

$$
\begin{aligned}
& \dot{Q}=P_{I}+L \frac{Q_{2}}{2} \\
& \dot{Q}_{2}=P_{2}-L \frac{Q_{1}}{2} .
\end{aligned}
$$

Then the Lagrangian is

$$
\begin{gathered}
I=\sum_{i} P_{i} \dot{Q}_{i}-H \text { or } \\
I=\frac{I}{2}\left(\dot{Q}_{1}{ }^{2}+\dot{Q}_{2}{ }^{2}\right)+\frac{1}{2} L\left(Q_{1} \dot{Q}_{2}-Q_{2} \dot{Q}_{1}\right)+\frac{I}{2}\left(\frac{L^{2}}{4}-\Omega^{2}\right)\left(Q_{1}{ }^{2}+Q_{2}{ }^{2}\right) .
\end{gathered}
$$


Note that the gyroscopic term in the Lagrangian formulation is linear in the time derivative of a coordinate. In the text I have been somewhat cavalier in labeling the complications arising from all linear time derivatives as "gyroscopic". Following the procedure to go to the Euclidean formulation in Equation (79), we find for the Euclidean Lagrangian,

$$
L_{E}=\frac{1}{2}\left(\dot{Q}_{1}{ }^{2}+\dot{Q}_{2}^{2}\right)-\frac{i L}{2}\left(Q_{1} \dot{Q}_{2}-Q_{2} \dot{Q}_{1}\right)-\frac{I}{2}\left(\frac{L^{2}}{4}-\Omega^{2}\right)\left(Q_{1}{ }^{2}+Q_{2}{ }^{2}\right) \text {. }
$$

The dot above now implies a $\tau$ derivative rather than a $t$ derivative. The second variation of the Euclidean Lagrangian is then

$$
\delta^{2} I_{E}=\left(\begin{array}{cc}
-\frac{\partial^{2}}{\partial \tau^{2}}+\Omega^{2}-\frac{L^{2}}{4} & -i L \frac{\partial}{\partial \tau} \\
+i L \frac{\partial}{\partial \tau} & -\frac{\partial^{2}}{\partial \tau^{2}}+\Omega^{2}-\frac{L^{2}}{4}
\end{array}\right)
$$

If we now examine one Fourier mode of our quantum fluctuation, or in effect replace $Q$ by $q_{K} e^{i k \tau}$, then, diagonalizing the above matrix and requiring positivity of its eigenvalues, we get

$$
\left[K^{2}+\Omega^{2}-L^{2} / 4-\lambda\right]^{2}+K^{2} L^{2}=0
$$

or in general

$$
\begin{gathered}
\operatorname{det}\left(k^{2}+G^{2}+V-\lambda I+2 k G\right)=0 \\
\lambda>0 .
\end{gathered}
$$

This can then be compared to Equation (113) for gyroscopic stability and Equation (115) for energy minimality. Proceeding we now get from Equation (127) 


$$
\lambda=K^{2}+\Omega^{2}-L^{2} / 4 \pm i K L
$$

For energy minimality we required that

$$
\Omega^{2}>L^{2} / 4,
$$

while for gyroscopic stability

$$
\Omega^{2}>0
$$

Comparing the above equations to Equation (81) and the arguments in the text, we see that except for the addition of an imaginary part to $\lambda$, the arguments go through as before. If we are expanding around a minimum of the energy, then $\operatorname{Re} \lambda>0$. Also, if $\operatorname{Re} \lambda<0$, then we are not at a minimum of the energy. In this case, however, although the one loop effective action is ill-defined or has an imaginary part, the system may be classically, gyroscopically, stable. (This is indeed the situation for the case of the vertical top, since $\Omega^{2}-L^{2} / 4=\mathrm{Mgl}$. If $\kappa^{2}<\mathrm{Mgl}$, the real part of $\lambda$ is negative definite, and although the energy is not a minimum, the system is gyroscopically stable.) The appearance of an imaginary contribution to $\lambda$ is not a problem for the evaluation of the functional integral since

$$
\int_{-\infty}^{\infty} d x e^{(-a \pm i b) x^{2}}=\left(\frac{\pi}{a \pm i b}\right)^{1 / 2}
$$

if $a>0$. 
B. 4. Yang-Mills Fields

In Reference (47), Jackiw and Rossi continue the stability analysis for Yang-Mills fields. Complications arise because Gauss's Law must be imposed as a constraint rather than as a consequence of Hamilton's equations. Nevertheless, for the Yang-Mills theory in the presence of an extended static source, it is demonstrated that the stability equations are in the same form as in Equation (112) with

$$
\begin{aligned}
& G=-\delta^{i j} g c{ }^{a b c_{A_{0}} b} \delta\left(\vec{r}-\vec{r}^{\prime}\right) \\
& V=\left\{\varepsilon^{i K m_{D_{m}} c b_{D_{n}} b a_{\varepsilon} n K j}-\varepsilon^{i K j} g c c b a_{B}{ }_{K}\right\}
\end{aligned}
$$

(See also Equation (92).)

The canonical coordinates for this theory are the vector potentials $\mathrm{A}^{i}$ and the canonical momenta are the electric fields $\mathrm{E}^{i} \mathrm{a}$. The magnetic fields, $\mathrm{B}^{i} \mathrm{a}$, are defined as in Equation (45). Note that it is the presence of non-vanishing components of $\mathrm{A}^{0}$ a that produce the gyroscopic term in Equation (130). In this case it is the source which plays the same role as the cyclic variable $L$ in the top problem. The source $\mathrm{J}_{a}^{\circ}=\rho_{a}$ is the generator of gauge transformations or color rotations, and is thus a color angular momentum (isospin). If we fix $\rho_{a}$ to be in a particular direction in color space, this is analogous to defining the symmetry axis in the top problem. In Reference (47) this direction is labeled the "electromagnetic" direction, while orthogonal directions are labeled "charged". As in the top problem, the gyroscopic 
74

term only affects the "charged" direction. This is so for the Yang-Milis problem, because the covariant conservation law for the source forces $A_{a}^{0}$ to be parallel to the source. For the case $\rho_{a}=\delta_{a 3} q$, the "Abelian Coulomb" case, Jackiw and Ross explicitly demonstrate the existence of "charged" fluctuations which lower the energy below that of the stable Coulomb solution, indicating that this is indeed an example of gyroscopic stability. 


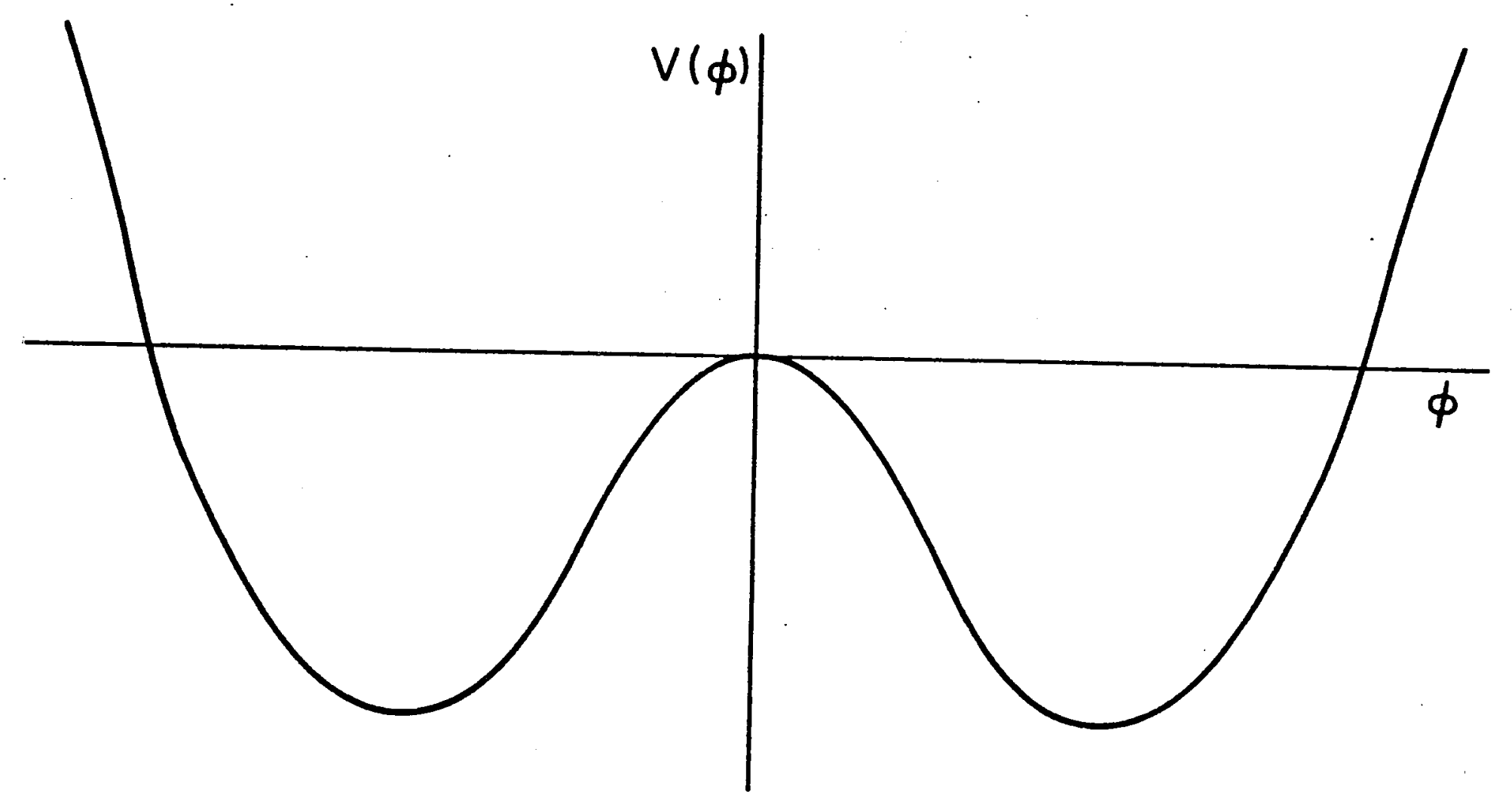

Figure 1. Quartic Potential, $V(\phi)$, with a negative mass squared versus $\phi$.

vt 


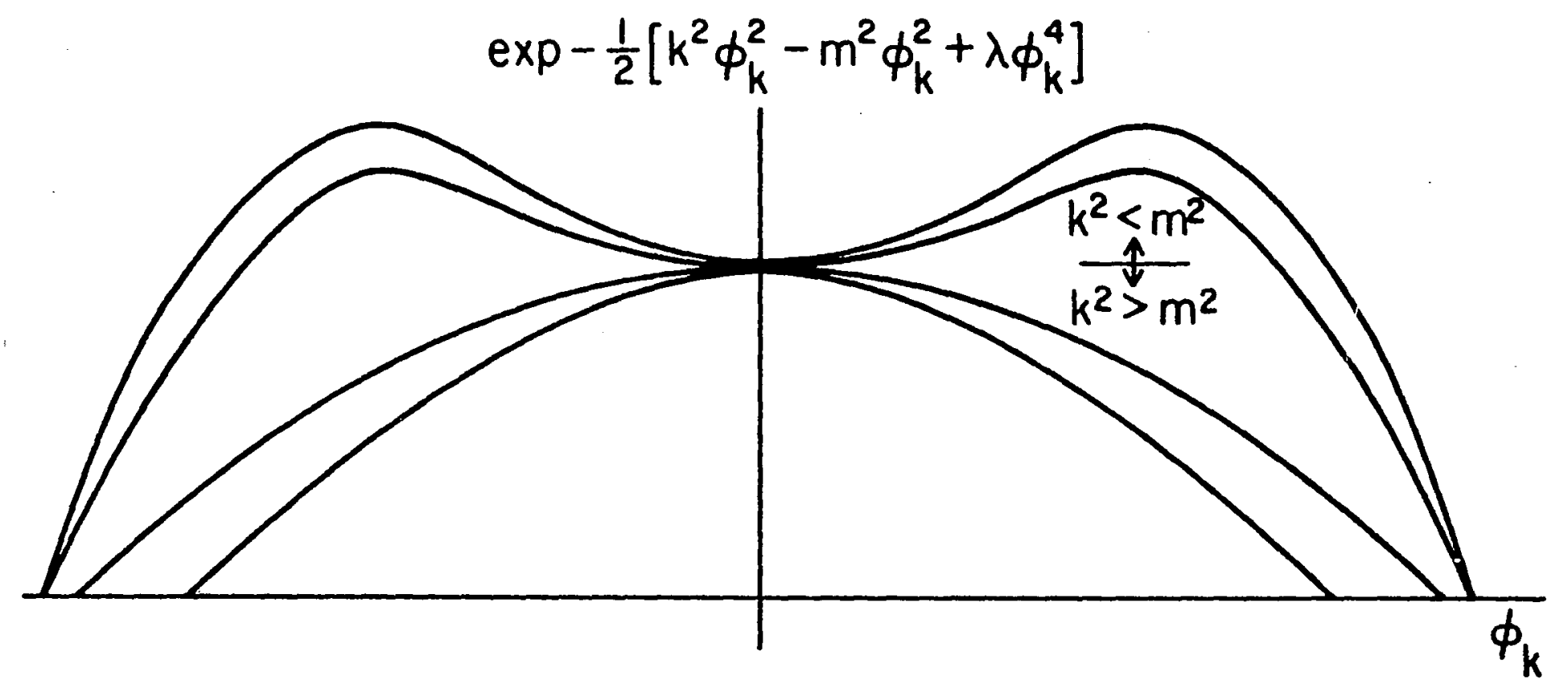

Figure 2. Integrand of minus the Euclidean action versus the field modes, $\phi_{\mathrm{k}}$, for various values of $\mathrm{k}^{2}$. 


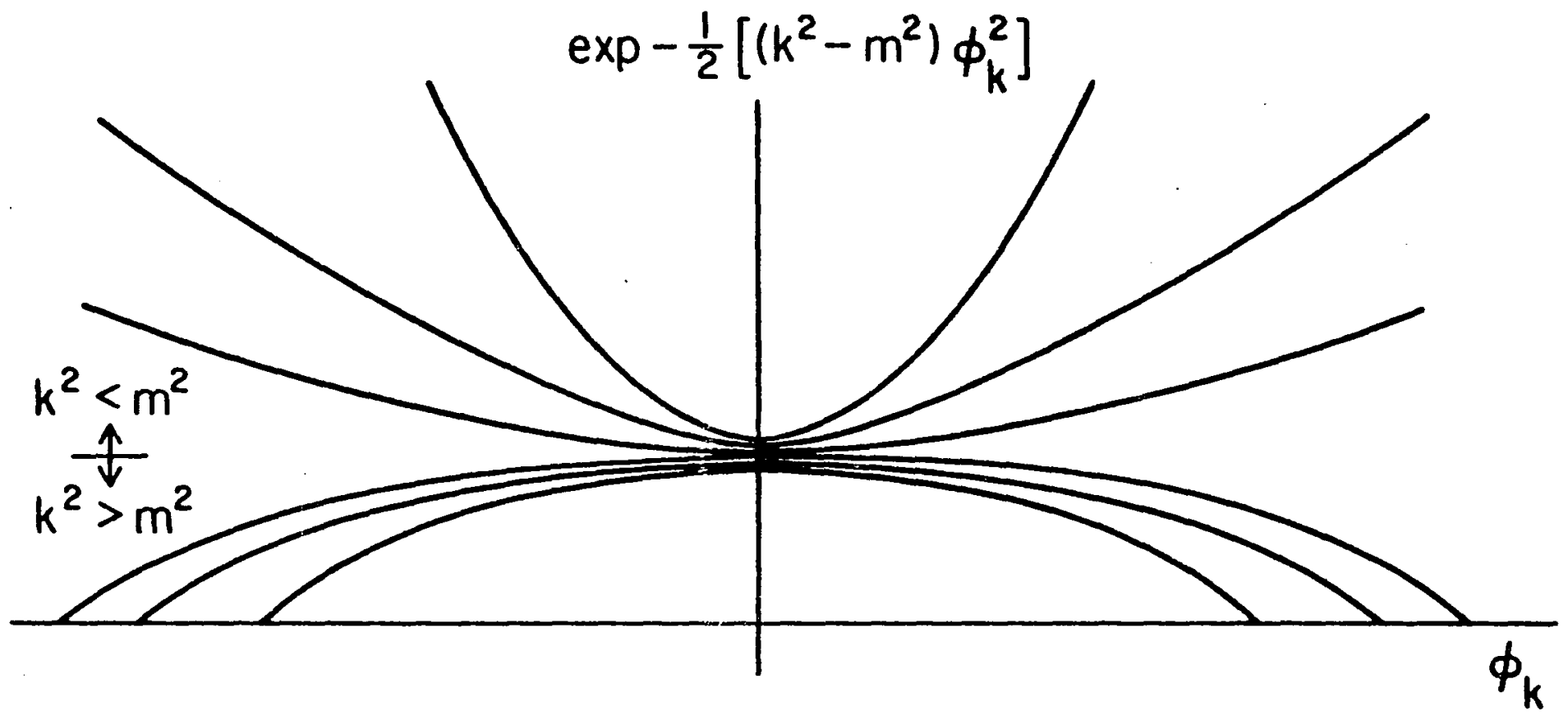

Figure 3. Quadratic approximations to the integrands which appear in Figure 2. 


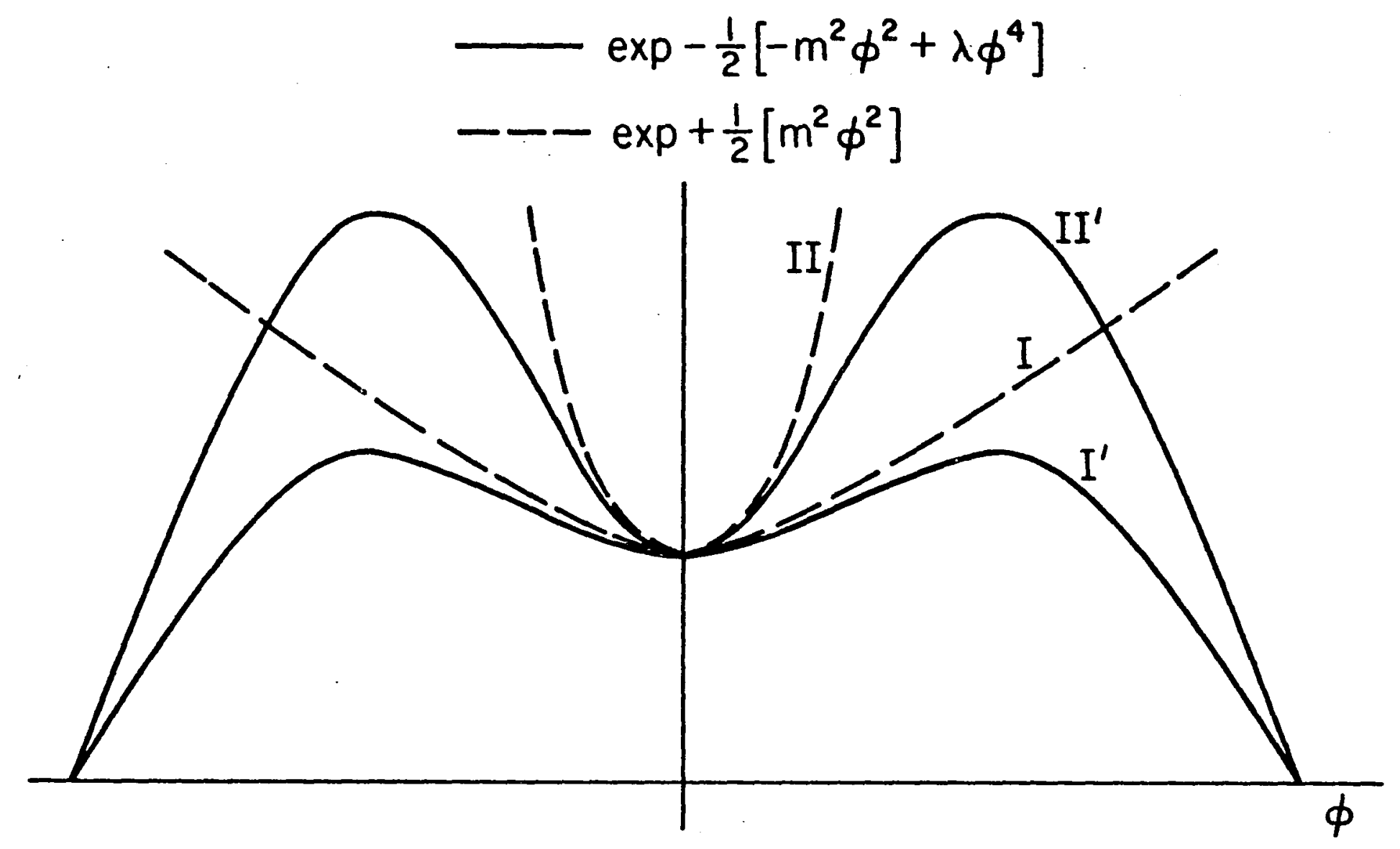

Figure 4. Solid lines - Euclidean functional integrand for $k^{2}=0$ versus field modes, $\phi$, for $m_{I I}^{\prime}>m_{I}^{\prime}$. Dashed lines -- Quadratic approximation to the solid lines where $m_{I I}=m_{I I}^{\prime} m_{I}=m_{I}^{\prime}$. 

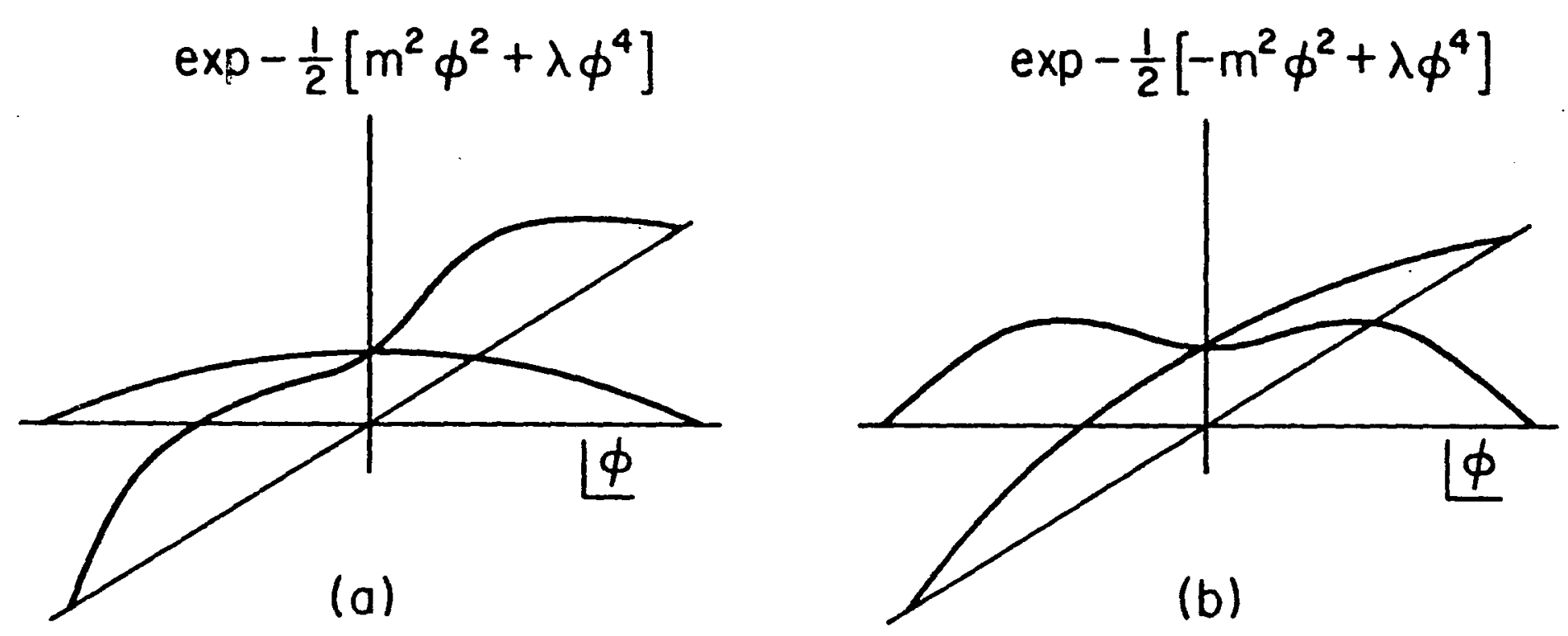

Figure sa. Euclidean functional integrand for $k^{2}=0$ and positive mass squared in the complex field mode plane.

Figure sb. Same as in Figure $5 b$ except for negative mass squared.

ธี 


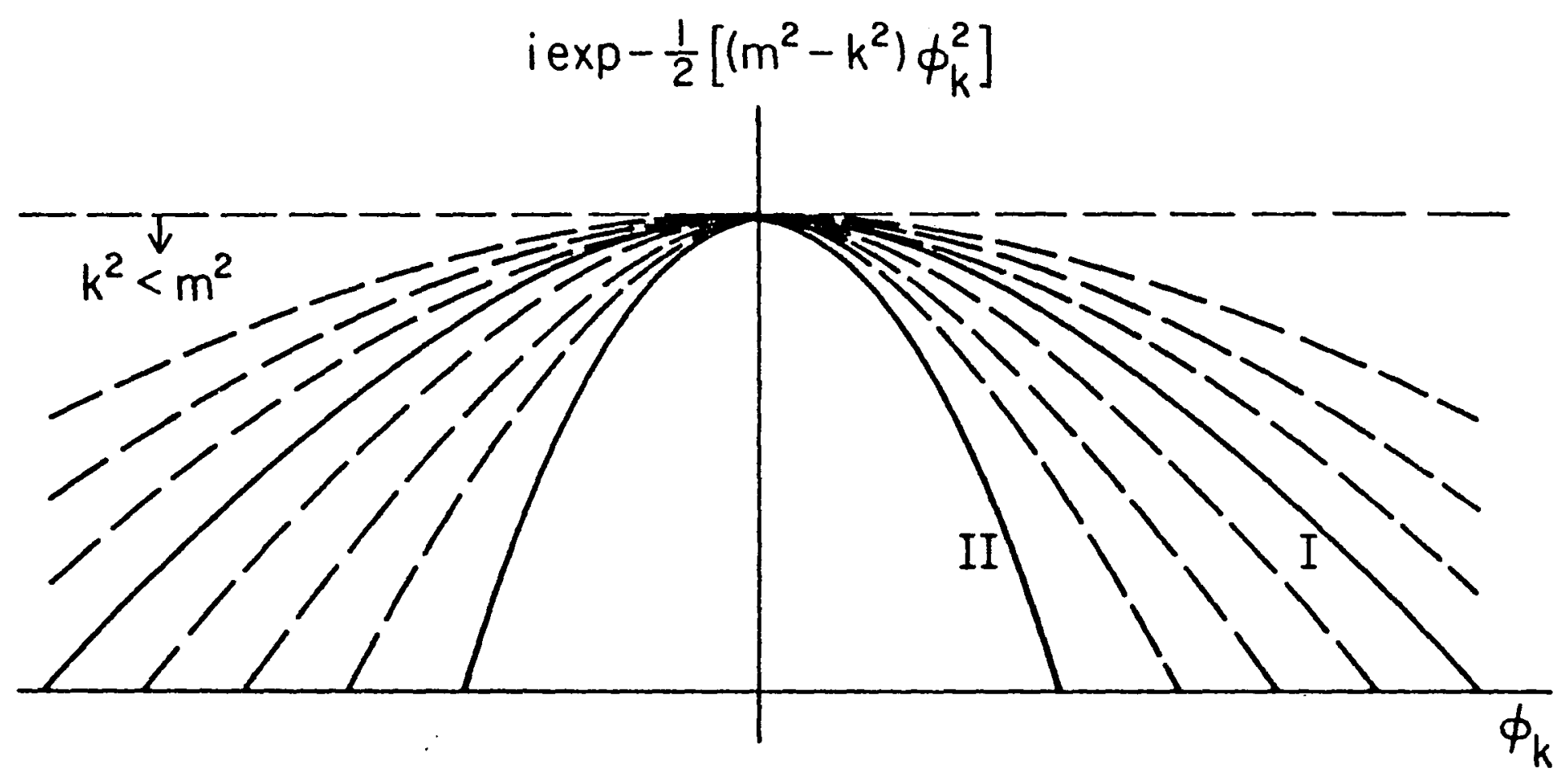

Figure 6. Analytic continuation of the functional integrand represented in Figure 3 for various values of $\mathrm{k}^{2}<\mathrm{m}^{2}$ where $\mathrm{m}_{\mathrm{II}}>\mathrm{m}_{\mathrm{I}}$. 


\section{LIST OF REFERENCES}


1. S. Coleman, "Secret Symmetries" in Laws of Hadronic Matter, ed. A. Zichichi (Academic Press, New York and London, 1975) and $G$. Jona-Lasino, Nuovo Cimento 34, 1790 (1964).

2. S. Mandelstam, "General Introduction to Confinement" in Proceedings of Les Houches Winter meeting, "Common Trends in Particle and Condensed Matter Physics" - 1980.

3. B. Dewitt, Dynamical Theory of Groups and Fields, in Relativity, Groups, and Topology, Les Houches (Gordon and Breach, New York, 1964).

4. B. Zumino, Effective Lagrangians and Broken Symmetries, in Lectures on Elementary Particles and Quantum Field Theory, [The M.I.T. Press, Cambridge and London, 1970].

5. R. Jackiw, Phys. Rev. D 10, 2491 (1974).

6. J. Schwinger, Phys. Rev. 82, 664 (1951).

7. S. Weinberg, Phys. Rev. D I, 2887 (1973).

8. W. Dittrich, Fortschr. Phys. 26, 289 (1978).

9. R. Fukuda and T. Kugo, Phys. Rev. D 13, 3469 (1976).

10. B. S. Dewitt, Phys. Rev. 162, 1195, 1239 (1967).

11. J. Honerkamp, Nucl. Phys. B 48, 269 (1972).

12. I. Ya Arefieva, L. D. Faddeev, A. A. Slavnov, Theo. Math. Phys. 21, 1165 (1974).

13. R. Kallosh, Nucl. Phys. B 78, 293 (1974).

14. G. 't Hooft, Nucl. Phys. B 62, 444 (1973).

15. G. 't Hooft, The Background Field Method in Gauge Field Theories, in Functional and Probabilistic Methods in Quantum Field Theory (Wroclaw University, Wroclaw, 1976).

16. N. K. Nielsen and P. Olesen, Nucl. Phys. B 144, 376 (1978). H. Flyvbjerg, Niels Bohr Institute, Preprint No. NBI-HE-80-16 $(1980)$.

17. J. Ambjorn and P. Olesen, Niels Bohr Institute Preprint No. NBI-HE-80-14 (1980).

18. H. Falomir and F. Schaposnik, Phys. Rev. D 21, 2859 (1980).

19. G. K. Savvidy, Phys. Lett. 71 B, 133 (1977). 
20. S. G. Matinyan and G. K. Savvidy, Nucl. Phys. B 134, 539 (1978).

21. I. A. Batalin, S. G. Matinyan, and G. K. Savvidy, Yad. Fiz. 26, 407 (1977) [Sov. J. Nucl. Phys. 26, 214 (1977)].

22. A. Yildiz and P. H. Cox, Phys. Rev. D 21, 1095 (1980) and A. Yildiz and P. H. Cox, Harvard University Preprint No. HUTP-79/AO 32 .

23. M. J. Duff and M. Ramón-Medrano, Phys. Rev. D 12, 3357 $(1975)$.

24. A. Salam and J. Strathdee, Nucl. Phys. B 90, 209 (1975).

25. S. Weinberg, Phys. Rev. D $\underline{9}, 3357$ (1974).

26. I. Dolan and R. Jackiw, Phys. Rev. D 9, 3320 (1974).

27. N. K. Nielsen and P. Olesen, Phys. Lett. 79 B, 304 (1978).

28. J. Ambjorn, N. K. Nielsen and P. Olesen, Nucl. Phys. B 152,75 (1979).

29. H. B. Nielsen and P. Olesen, Nucl. Phys. B 160, 380 (1979).

30. H. B. Nielsen and M. Ninomiya, Nucl. Phys. B 156, I (1979).

31. J. Ambjorn and P. Olesen, Niels Bohr Institute Preprint No. NBI-HE-79-38; H. B. Nielsen and P. Olesen, Niels Bohr Institute Preprint No. NBI-HE-79-45; H. B. Nielsen and M. Ninomiya, Niels Bohr Institute Preprint No. NBI$\mathrm{HE}-79-43$.

32. W-Y. Tsai and A. Yildiz, Phys. Rev. D 4, 3643 (1971); T. Goldman, $W-Y$. Tsai and A. Yildiz, Phys. Rev. D 5 , 1926 (1972); T. Goldman, SLAC-PUB-1363 (1973).

33. P. Sikivie, Phys. Rev. D 20, 877 (1979).

34. S. J. Chang and N. Weiss, Phys. Rev. D 20869 (1979).

35. G. Cosenza and F. Neri, New York University Preprint No. NYU/TR $3 / 80$.

36. S. Coleman, Phys. Rev. D 15, 2929 (1977); S. C. Callan and S. Coleman, Phys. Rev. D 16, 1762 (1977).

37. S. Coleman and F. Deluccia, SLAC Preprint No. SLAC-PUB$2463(1980)$. 
38. J. S. Langer, Ann. Phys. (N.Y.) 41, 108 (1967).

39. R. Jackiw, M.I.T., C.T.P. No. 816 (1979).

40. S. Abers and B. W. Lee, Phys. Rep. I, I (1973).

41. W. Fischler and R. Brout, Phys. Rev. D 11, 905 (1975).

42. S. Mandelstam, U.S. Berkeley Preprint No. UCB-PTH-79/8 (1979).

43. G. 't Hooft, Nucl. Phys. B 153, 141 (1979).

44. V. Vanyashin and M. Terent'ev, JETP 21, 375 (1965).

45. H. Pagels and E. Tomboulis, Nucl. Phys. B 143, 485 (1978).

46. D. G. Gross and F. Wilczek, Phys. Rev. D 8, 3633 (1973);

D. G. Gross and F. Wilczek, Phys. Rev. D $\underline{\underline{9}}, 980$ (1974).

47. R. Jackiw and P. Rossi, Phys. Rev. D 21, 425 (1980).

48. P. Sikivie and N. Weiss, Phys. Rev. D 20, 487 (1979).

49. M. Magg, Nucl. Phys. B 158, 154 (1979).

50. H. Arodz, Phys. Lett. B 84, 524 (1979).

5I. R. Jackiw and A. Kerman, M.I.T., C.T.P. No. 763 (1979).

52. L. D. Landau and E. M. Lifshitz, Quantum Mechanics (Pergamon Press, New York, 1977) pp. 456-458.

53. S. Coleman and E. Weinberg, Phys. Rev. D I, 1888 (1973).

54. H. Goldstein, Classical Mechanics (Addison-Wesley, Reading, Massachusetts; London, England, 1950).

55. B. J. Harrington, private communication (1980). 